\title{
Expansion of rational numbers in Möbius number systems
}

\author{
Petr Kůrka \\ Center for Theoretical Study, \\ Academy of Sciences and Charles University in Prague, \\ Jilská 1, CZ-11000 Praha 1, Czechia
}

December 28, 2009

\begin{abstract}
Möbius number systems represent real numbers by symbolic sequences of Möbius transformations. We say that a Möbius number system is rational, if it represents rational numbers by periodic sequences, and we give some criteria for this property.
\end{abstract}

\section{Introduction}

Möbius number systems introduced in Kůrka [3, 6] generalize both positional number systems and continued fractions. They represent real numbers by sequences of Möbius transformations. A Möbius iterative system consists of real Möbius transfromations $\left(F_{a}: \overline{\mathbb{R}} \rightarrow \overline{\mathbb{R}}\right)_{a \in A}$ indexed by a finite alphabet $A$, which act on the extended real line $\overline{\mathbb{R}}=\mathbb{R} \cup\{\infty\}$. The convergence space $\mathbb{X}_{F}$ of $F$ consists of infinite words $u \in A^{\mathbb{N}}$ such that the sequence $F_{u_{0}} F_{u_{1}} \cdots F_{u_{n}}(z)$ converges to a real number $\Phi(u) \in \overline{\mathbb{R}}$, whenever $z$ belongs to the upper half of the complex plane. If $\Sigma \subset \mathbb{X}_{F}$ is a subshift such that $\Phi: \Sigma \rightarrow \mathbb{X}_{F}$ is continuous and surjective, then we say that $(F, \Sigma)$ is a Möbius number system. In Kưrka and Kazda [7] we use an interval cover or almost-cover $\mathcal{W}=\left(W_{a}\right)_{a \in A}$ of $\overline{\mathbb{R}}$ to construct expansions of real numbers. A number $x \in \overline{\mathbb{R}}$ is expanded into its representation $u \in A^{\mathbb{N}}$ with $\Phi(u)=x$, if $x \in W_{u_{0}}, F_{u_{0}}^{-1}(x) \in W_{u_{1}}$, etc. The set of all these expansions forms the expanding subshift $\mathcal{S}_{\mathcal{W}} \subset A^{\mathbb{N}}$. We show that $\left(F, \mathcal{S}_{\mathcal{W}}\right)$ is a Möbius number system, provided the expansion quotient $\mathbf{Q}(\mathcal{W})$ of $\mathcal{W}$ is larger than one.

In the present paper we study expansions of rational numbers in the integer Möbius number systems, whose transformations have integer entries. We say that an integer Möbius number system is rational, if every rational number has a periodic expansion. We define the rational expansion interval $\mathbf{R}(M)$ of a transformation $M$ and show that the system is rational provided $\left(\mathbf{R}\left(F_{a}\right)\right)_{a \in A}$ is an almost-cover of $\overline{\mathbb{R}}$, i.e., provided the closures $\overline{\mathbf{R}\left(F_{a}\right)}$ cover $\overline{\mathbb{R}}$. Using this criterion, we can show the rationality of a large class of Möbius number systems. To show that some systems are not rational, we consider their dynamics in the residue classes modulo certain primes. Using this tool we analyze the cancellations which occur during expansions and show that some systems are not rational because the norm of rational numbers steadily grows during their expansion.

We apply our theory to hyperbolic rectangular systems whose fixed points are numbers $0,1, \infty,-1$ and show that the square systems with the same expansion quotients in the horizontal and vertical directions are never rational, while some non-square rectangular systems are. Then we turn to modular systems whose transformations have unit determinant. In modular systems the condition of rationality coincides with the condition of convergence, so naturally defined modular systems $\left(F, \Sigma_{\mathcal{W}}\right)$ are usually rational. Modular systems yield nice representations of rational numbers which are related to continued fractions and to the Farey fractions. On the other hand, they are never redundant, and therefore unsuitable for arithmetic algorithms.

Finaly we consider three bimodular octanic systems consisting of eight transformations with determinant two, which optimize the almost-cover $\left(\mathbf{R}\left(F_{a}\right)\right)_{a \in A}$ and have nice symmetries. Besides being rational, these systems are redundant, and therefore support the arithmetical algorithms for the exact real computation decribed for example in Vuillemin [10] and generalized to Möbius number systems by Kưrka and Kazda [7]. 


\section{Möbius transformations}

The extended real line $\overline{\mathbb{R}}=\mathbb{R} \cup\{\infty\}$ can be regarded as a projective space, i.e., the space of onedimensional subspaces of the two-dimensional vector space. On $\overline{\mathbb{R}}$ we have homogenous coordinates $x=\left(x_{0}, x_{1}\right) \in \mathbb{R}^{2} \backslash\{(0,0)\}$ with equality $x=y$ iff $x_{0} y_{1}=x_{1} y_{0}$. We regard $x \in \overline{\mathbb{R}}$ as a column vector, and write it usually as $x=x_{0} / x_{1}$, for example $\infty=1 / 0$. For distinct $a, b \in \mathbb{R}$, the open interval $(a, b)$ is the set $\{x \in \mathbb{R}: a<x<b\}$ if $a<b$ and $\{x \in \mathbb{R}: a<x$ or $x<b\} \cup\{\infty\}$ if $b<a$. For $x \in \mathbb{R}$ we have $x \in(a, b)$ iff $(a-x)(x-b)(b-a)>0$. In homogenous coordinates we get a formula which works for all distinct $a, b \in \overline{\mathbb{R}}:(a, b)=\left\{x \in \overline{\mathbb{R}}:\left(a_{0} x_{1}-a_{1} x_{0}\right)\left(x_{0} b_{1}-x_{1} b_{0}\right)\left(b_{0} a_{1}-b_{1} a_{0}\right)>0\right\}$. The map $\mathbf{d}(z)=(i z+1) /(z+i)$ maps $\overline{\mathbb{R}}$ to the unit circle $\partial \mathbb{D}=\{z \in \mathbb{C}:|z|=1\}$ and the upper half-plane $\mathbb{U}=\{z \in \mathbb{C}: \Im(z)>0\}$ conformally to the unit disc $\mathbb{D}=\{z \in \mathbb{C}:|z|<1\}$. The circle distance $\varrho(x, y)$ of $x, y \in \overline{\mathbb{R}}$ is the length of the shortest arc joining $\mathbf{d}(x)$ and $\mathbf{d}(y)$ in $\partial \mathbb{D}$ :

$$
\varrho(x, y)=2 \arcsin \frac{|x-y|}{\sqrt{\left(x^{2}+1\right)\left(y^{2}+1\right)}}=2 \arcsin \frac{\left|x_{0} y_{1}-x_{1} y_{0}\right|}{\|x\| \cdot|| y||},
$$

where $\|x\|=\sqrt{x_{0}^{2}+x_{1}^{2}}$ is the euclidean norm of $x \in \mathbb{R}^{2}$. The length of an open interval $B_{r}(a)=\{x \in$ $\overline{\mathbb{R}}: \varrho(x, a)<r\}$ is $\left\|B_{r}(a)\right\|=2 r$ provided $r<\pi$.

A real orientation-preserving Möbius transformation (MT) is a self-map of $\overline{\mathbb{R}}$ of the form $M_{(a, b, c, d)}(x)=\frac{a x+b}{c x+d}=\frac{a x_{0}+b x_{1}}{c x_{0}+d x_{1}}$ where $a, b, c, d \in \mathbb{R}$ and $\operatorname{det}\left(M_{(a, b, c, d)}\right)=a d-b c>0$. MT act also on the upper half-plane $\mathbb{U}$. On $\overline{\mathbb{D}}:=\mathbb{D} \cup \partial \mathbb{D}$ we get disc Möbius transformations $\widehat{M}$ defined by $\widehat{M}_{(a, b, c, d)}(z)=\mathbf{d} \circ M_{(a, b, c, d)} \circ \mathbf{d}^{-1}(z)=(\alpha z+\beta) /(\bar{\beta} z+\bar{\alpha})$, where $\alpha=(a+d)+(b-c) i, \beta=(b+c)+(a-d) i$. Define the norm of a Möbius transformation $M=M_{(a, b, c, d)}$ by $\|M\|:=\left(a^{2}+b^{2}+c^{2}+d^{2}\right) /(a d-b c)$. We have $\|M\| \geq 2$ for each $M$, and $\|M\|=2$ iff $M$ is a rotation, i.e., if $M=R_{\alpha}=M_{\left(\cos \frac{\alpha}{2}, \sin \frac{\alpha}{2},-\sin \frac{\alpha}{2}, \cos \frac{\alpha}{2}\right)}$ for some $\alpha$. The circle derivation, the expansion interval and the expansion quotient of $M$ are defined by

$$
\begin{aligned}
M^{\bullet}(x) & :=\lim _{y \rightarrow x} \frac{\varrho(M(y), M(x))}{\varrho(y, x)}=\left|\widehat{M}^{\prime}(\mathbf{d}(x))\right|=\frac{\operatorname{det}(M) \cdot\|x\|^{2}}{\|M(x)\|^{2}} \\
\mathbf{V}(M) & :=\left\{x \in \overline{\mathbb{R}}:\left(M^{-1}\right)^{\bullet}(x)>1\right\}, \\
\mathbf{Q}(M) & :=\max \left\{M^{\bullet}(x): x \in \overline{\mathbb{R}}\right\}=\frac{1}{2}\left(\| M||+\sqrt{\|M\|^{2}-4}\right)=\frac{1+|\widehat{M}(0)|}{1-|\widehat{M}(0)|}
\end{aligned}
$$

We have $(M N)^{\bullet}(x)=M^{\bullet}(N(x)) \cdot N^{\bullet}(x)$. If $M$ is a rotation, then $M^{\bullet}(x)=1$ and $\mathbf{V}(M)$ is empty. Otherwise $\mathbf{V}(M)$ is a nonempty interval. The square of the trace of $M$ is $\operatorname{tr}^{2}\left(M_{(a, b, c, d)}\right)=(a+d)^{2} /(a d-$ $b c)$. An MT is hyperbolic if $\operatorname{tr}^{2}(M)>4$, parabolic if $\operatorname{tr}^{2}(M)=4$ and elliptic if $\operatorname{tr}^{2}(M)<4$. A hyperbolic MT has a stable fixed point $s_{M} \in \overline{\mathbb{R}}$ with $M^{\bullet}\left(s_{M}\right)<1$ and un unstable fixed point $u_{M} \in \overline{\mathbb{R}}$ with $M^{\bullet}\left(u_{M}\right) \cdot M^{\bullet}\left(s_{M}\right)=1$. A parabolic MT has a unique fixed point $s_{M}$ with $M^{\bullet}\left(s_{M}\right)=1$. An elliptic MT has no fixed point in $\overline{\mathbb{R}}$.

\section{Möbius number systems}

For a finite alphabet $A$ denote by $A^{*}:=\bigcup_{m \geq 0} A^{m}$ the set of finite words and by $A^{+}:=A^{*} \backslash\{\lambda\}$ the set of finite non-empty words. The length of a word $u=u_{0} \ldots u_{m-1} \in A^{m}$ is $|u|:=m$. We denote by $A^{\mathbb{N}}$ the Cantor space of infinite words equipped with the metric $d(u, v):=2^{-k}$, where $k=\min \left\{i \geq 0: u_{i} \neq v_{i}\right\}$. We say that $v \in A^{*}$ is a subword of $u \in A^{*} \cup A^{\mathbb{N}}$ and write $v \sqsubseteq u$, if $v=u_{[i, j)}=u_{i} \ldots u_{j-1}$ for some $0 \leq i \leq j \leq|u|$. The cylinder of $u \in A^{n}$ is the set $[u]:=\left\{v \in A^{\mathbb{N}}: v_{[0, n)}=u\right\}$. Given $u \in A^{m}, v \in A^{p}$, denote by $u . v \in A^{\mathbb{N}}$ the periodic word with preperiod $u$ and period $v$ defined by $(u \cdot v)_{i}=u_{i}$ for $i<n$ and $(u \cdot v)_{n+k m+i}=v_{i}$ for $i<m, k \geq 0$. The set of periodic words is denoted by $\mathbb{P}_{A}:=\left\{u \in A^{\mathbb{N}}: \exists m \geq\right.$ $\left.0, \exists p>0, \sigma^{m+p}(u)=\sigma^{m}(u)\right\}$, where the shift map $\sigma: A^{\mathbb{N}} \rightarrow A^{\mathbb{N}}$ is defined by $\sigma(u)_{i}=u_{i+1}$. A subshift is a nonempty set $\Sigma \subseteq A^{\mathbb{N}}$ which is closed and $\sigma$-invariant, i.e., $\sigma(\Sigma) \subseteq \Sigma$. For a subshift $\Sigma$ there exists a set $D \subseteq A^{+}$of forbidden words such that $\Sigma=\Sigma_{D}:=\left\{x \in A^{\mathbb{N}}: \forall u \sqsubseteq x, u \notin D\right\}$. A subshift is uniquely determined by its language $\mathcal{L}(\Sigma):=\left\{u \in A^{*}: \exists x \in \Sigma, u \sqsubseteq x\right\}$. A labelled graph over $A$ is a structure $G=(V, E, s, t, l)$, where $V=|G|$ is the set of vertices, $E$ is the set of edges, $s, t: E \rightarrow V$ are the source and target maps, and $l: E \rightarrow A$ is a labelling function. The subshift $\Sigma_{G} \subseteq A^{\mathbb{N}}$ of $G$ consists of all labels of paths of $G$. A subshift is sofic, if it is the subshift of a finite labelled graph. A subshift is of finite type (SFT), if the set $D$ of its forbidden words is finite (see Lind and Marcus [8]). 
An iterative system is a continuous map $F: A^{*} \times X \rightarrow X$, or a family of continuous maps $\left(F_{u}: X \rightarrow X\right)_{u \in A^{*}}$ on a compact metric space $X$ satisfying $F_{u v}=F_{u} \circ F_{v}$, and $F_{\lambda}=$ Id. It is determined by generators $\left(F_{a}: X \rightarrow X\right)_{a \in A}$. Topological dynamics of Möbius iterative systems has been investigated in Kůrka [5].

Definition 1 (Kůrka [6]) We say that $F: A^{*} \times \overline{\mathbb{R}} \rightarrow \overline{\mathbb{R}}$, is a Möbius iterative system, if all $F_{a}: \overline{\mathbb{R}} \rightarrow \overline{\mathbb{R}}$ are orientation-preserving Möbius transformations. The convergence space $\mathbb{X}_{F} \subseteq A^{\mathbb{N}}$ and the symbolic representation $\Phi: \mathbb{X}_{F} \rightarrow \overline{\mathbb{R}}$ are defined by

$$
\begin{aligned}
\mathbb{X}_{F} & :=\left\{u \in A^{\mathbb{N}}: \lim _{n \rightarrow \infty} F_{u_{[0, n)}}(i) \in \overline{\mathbb{R}}\right\}, \\
\Phi(u) & :=\lim _{n \rightarrow \infty} F_{u_{[0, n)}}(i),
\end{aligned}
$$

where $i \in \mathbb{U}$ is the imaginary unit. If $\Sigma \subseteq \mathbb{X}_{F}$ is a subshift such that $\Phi: \Sigma \rightarrow \overline{\mathbb{R}}$ is continuous and surjective, then we say that $(F, \Sigma)$ is a Möbius number system. We say that a Möbius number system is redundant, if for every continuous map $g: \overline{\mathbb{R}} \rightarrow \overline{\mathbb{R}}$ there exists a continuous map $f: \Sigma \rightarrow \Sigma$ such that $\Phi f=g \Phi$. An interval almost-cover for $F$ is a family of open intervals $\mathcal{W}=\left\{W_{a}: a \in A\right\}$ such that $\bigcup_{a \in A} \overline{W_{a}}=\overline{\mathbb{R}}$. If $W_{a}$ are mutually disjoint, we say that $\mathcal{W}$ is an interval partition. The expansion subshift $\mathcal{S}_{\mathcal{W}}$ of $\mathcal{W}$ is defined by

$$
\begin{aligned}
& \mathcal{S}_{\mathcal{W}}=\left\{u \in A^{\mathbb{N}}: \forall k>0, W_{u_{[0, k)}} \neq \emptyset\right\}, \text { where } \\
& W_{u}=W_{u_{0}} \cap F_{u_{0}}\left(W_{u_{1}}\right) \cap F_{u_{[0,2)}}\left(W_{u_{2}}\right) \cap \cdots \cap F_{u_{[0, n)}}\left(W_{u_{n}}\right), u \in A^{n+1}
\end{aligned}
$$

If $u \in \mathbb{X}_{F}$, then $\Phi(u)=\lim _{n \rightarrow \infty} F_{u_{[0, n)}}(z)$ for every $z \in \mathbb{U}$, and $\mathbf{d} \Phi(u)=\lim _{n \rightarrow \infty} \widehat{F}_{u_{[0, n)}}(z)$ for every $z \in \mathbb{D}$ (see Kazda [2]). For $v \in A^{+}, u \in A^{\mathbb{N}}$ we have $v u \in \mathbb{X}_{F}$ iff $u \in \mathbb{X}_{F}$ and then $\Phi(v u)=F_{v}(\Phi(u)$ ), so $\mathbb{X}_{F}$ is dense in $A^{\mathbb{N}}$. If $u . v \in \mathbb{P}$ is a periodic word with period $u$, then $u . v \in \mathbb{X}_{F}$ iff $F_{v}$ is not elliptic. Redundancy is necessary for the existence of exact real arithmetical algorithms (see Vuillemin [10] or Kůrka and Kazda [7]).

Proposition 2 (Kůrka and Kazda [7]) Let $\mathcal{W}$ be an interval almost-cover for $F$ and assume that for each $u \in A^{m}, a \in A$ we have $W_{u} \cap F_{a}^{-1}\left(W_{a}\right) \neq \emptyset \Rightarrow W_{u} \subseteq F_{a}^{-1}\left(W_{a}\right)$. Then $\mathcal{S}_{\mathcal{W}}$ is a SFT of order $m+1$.

If $B \subset A^{+}$is a finite set and $\mathcal{W}=\left\{W_{u}: u \in B\right\}$ an interval almost-cover, then we regard $B^{\mathbb{N}}$ as a subset of $A^{\mathbb{N}}$ and identify the subshift $\mathcal{S}_{\mathcal{W}} \subseteq B^{\mathbb{N}} \subset A^{\mathbb{N}}$ with the subshift of $A^{\mathbb{N}}$ defined by $\mathcal{S}_{\mathcal{W}, A}=\left\{\sigma^{n}(u): u \in \mathcal{S}_{\mathcal{W}}, n \geq 0\right\}$

Theorem 3 (Kůrka and Kazda [7]) Let $F$ be a Möbius iterative system and $B \subset A^{+}$a finite set such that $\mathcal{W}=\left\{\mathbf{V}\left(F_{u}\right): u \in B\right\}$ is an almost-cover of $\overline{\mathbb{R}}$. Then $\left(F, \mathcal{S}_{\mathcal{W}, A}\right)$ is a Möbius number system. If $\mathcal{W}$ is a cover of $\overline{\mathbb{R}}$, then $\left(F, \mathcal{S}_{\mathcal{W}, A}\right)$ is redundant.

We get a stronger condition if we evaluate circle derivations of $F_{u}^{-1}$ on their intervals $W_{u}$. Given a Möbius iterative system $F: A^{*} \times \overline{\mathbb{R}} \rightarrow \overline{\mathbb{R}}$ and an interval almost-cover $\mathcal{W}=\left\{W_{a}: a \in A\right\}$, define

$$
\begin{aligned}
\mathbf{q}(u) & :=\inf \left\{\left(F_{u}^{-1}\right)^{\bullet}(x): x \in W_{u}\right\} \\
\mathbf{Q}_{n}(\mathcal{W}) & :=\min \left\{\mathbf{q}(u): u \in \mathcal{L}\left(\mathcal{S}_{\mathcal{W}}\right) \cap A^{n}\right\} \\
\mathbf{Q}(\mathcal{W}) & :=\lim _{n \rightarrow \infty} \sqrt[n]{\mathbf{Q}_{n}(\mathcal{W})} \\
\mathbf{L}(\mathcal{W}) & :=\sup \left\{r>0: \forall x \in \overline{\mathbb{R}}, \exists a \in A, B_{r / 2}(x) \subseteq W_{a}\right\}
\end{aligned}
$$

where $B_{r / 2}(x)=\{y \in \overline{\mathbb{R}}: \varrho(y, x)<r / 2\}$. We have $\mathbf{Q}_{m+n}(\mathcal{W}) \geq \mathbf{Q}_{m}(\mathcal{W}) \cdot \mathbf{Q}_{n}(\mathcal{W})$, so the limit in $\mathbf{Q}(\mathcal{W})$ exists and $\mathbf{Q}(\mathcal{W}) \geq \sqrt[n]{\mathbf{Q}_{n}(\mathcal{W})}$ for each $n$. We call $\mathbf{Q}(\mathcal{W})$ the expansion quotient of $\mathcal{W}$ and $\mathbf{L}(\mathcal{W})$ the Lebesgue number of $\mathcal{W}$. The expansion quotient $\mathbf{Q}(\mathcal{W})$ expresses the rate of convergence of $\Phi$. For each $n$ we have $\max \left\{\left\|W_{u}\right\|: u \in \mathcal{L}\left(\mathcal{S}_{\mathcal{W}}\right) \cap A^{n}\right\} \leq 2 \pi / \mathbf{Q}_{n}(\mathcal{W})$. The Lebesgue number $\mathbf{L}(\mathcal{W})$ is a measure of redundancy of the system.

Theorem 4 (Kůrka and Kazda [7]) Let $F$ be a Möbius iterative system and $\mathcal{W}$ an interval almostcover for $F$. If $\mathbf{Q}_{n}(\mathcal{W}) \geq 1$ for some $n$, and if no $F_{u}$ with $u \in \mathcal{L}\left(\mathcal{S}_{\mathcal{W}}\right) \cap A^{n}$ is a rotation, then $\left(F, \mathcal{S}_{\mathcal{W}}\right)$ is a Möbius number system, and $\Phi\left([u] \cap \mathcal{S}_{\mathcal{W}}\right)=\overline{W_{u}}$ for each $u \in \mathcal{L}\left(\mathcal{S}_{\mathcal{W}}\right)$. If $\mathcal{W}$ is a cover of $\overline{\mathbb{R}}$, then $\left(F, \mathcal{S}_{\mathcal{W}}\right)$ is redundant. Conversely, if $\left(F, \mathcal{S}_{\mathcal{W}}\right)$ is a Möbius number system and if $\Phi\left([u] \cap \mathcal{S}_{\mathcal{W}}\right)=\overline{W_{u}}$ for each $u \in \mathcal{L}\left(\mathcal{S}_{\mathcal{W}}\right)$, then $\mathbf{Q}(\mathcal{W}) \geq 1$. 


\section{Rational Möbius number systems}

We investigate expansions of rational numbers in Möbius number system whose transformations have integer entries. To analyze the cancellations which occur during expansions, we work with the matrices which represent the transformations, rather than with the transformations themselves. Denote by $\mathbb{Z}$ the set of integers and by $\widehat{\mathbb{Q}}:=\mathbb{Z}^{2} \backslash\left\{\frac{0}{0}\right\}$. For $x=\frac{x_{0}}{x_{1}} \in \widehat{\mathbb{Q}}$ we denote by $\operatorname{gcd}(x)>0$ the greatest common divisor of $x_{0}, x_{1}$. Denote by $\overline{\mathbb{Q}}=\{x \in \widehat{\mathbb{Q}}: \operatorname{gcd}(x)=1\}$. Each rational number has two representations in $\overline{\mathbb{Q}}$. For each $m>0$ we have the map $\mathbf{d}_{m}: \widehat{\mathbb{Q}} \rightarrow \widehat{\mathbb{Q}}$ defined by $\mathbf{d}_{m}(x)=\frac{x_{0} / m}{x_{1} / m}$ if $m \operatorname{divides} \operatorname{gcd}(x)$, and $\mathbf{d}_{m}(x)=x$ otherwise. We have the map $\mathbf{d}: \widehat{\mathbb{Q}} \rightarrow \overline{\mathbb{Q}}$ defined by $\mathbf{d}(x)=\frac{x_{0} / \operatorname{gcd}(x)}{x_{1} / \operatorname{gcd}(x)}$. Denote by $\operatorname{gcd}\left(M_{(a, b, c, d)}\right)=\operatorname{gcd}(a, b, c, d)>0$ the greatest common divisor of the entries of a matrix $M \in G L(\mathbb{Z}, 2)$ with integer entries. We consider the subgroups

$$
\begin{aligned}
\widehat{\mathcal{M}} & =\{M \in G L(\mathbb{Z}, 2): \operatorname{det}(M)>0\}, \\
\mathcal{M} & =\{M \in \widehat{\mathcal{M}}: \operatorname{gcd}(M)=1\}, \\
\mathcal{M}_{p} & =\left\{M \in \widehat{\mathcal{M}}: \exists n \geq 0, \operatorname{det}(M)=p^{n}\right\},
\end{aligned}
$$

so $\mathcal{M}_{1}$ is the modular group, and we call $\mathcal{M}_{2}$ the bimodular group. We have a map $\mathfrak{d}: \widehat{\mathcal{M}} \rightarrow \mathcal{M}$ given by $\mathfrak{d}\left(M_{(a, b, c, d)}\right)=M_{(a / g, b / g, c / g, d / g)}$, where $g=\operatorname{gcd}(a, b, c, d)$. An MT $M \in \mathcal{M}$ acts on $\widehat{\mathbb{Q}}$ by multiplication $M_{(a, b, c, d)}(x)=\frac{a x_{0}+b x_{1}}{c x_{0}+d x_{1}}$. The composition $\mathbf{d} M: \overline{\mathbb{Q}} \stackrel{M}{\rightarrow} \widehat{\mathbb{Q}} \stackrel{\mathbf{d}}{\rightarrow} \overline{\mathbb{Q}}$ acts on $\overline{\mathbb{Q}}$.

Definition 5 We say that $F: A^{*} \times \overline{\mathbb{R}} \rightarrow \overline{\mathbb{R}}$ is an integer Möbius iterative system, if $F_{a} \in \mathcal{M}$ for each $a \in A$. We say that $F$ is rational, if every rational has a periodic expansion, i.e., if $\overline{\mathbb{Q}} \subseteq \Phi(\mathbb{P})$. We say that an integer Möbius number system $(F, \Sigma)$ is rational, if $\overline{\mathbb{Q}} \subseteq \Phi\left(\mathbb{P}_{A} \cap \Sigma\right)$. Given an interval almost-cover $\mathcal{W}=\left\{W_{a}: a \in A\right\}$ for $F$, define the labelled rational expansion graph with vertices $(x, s) \in \overline{\mathbb{Q}} \times\{-, 0,+\}$ and edges

$$
\begin{aligned}
& (x, s) \quad \stackrel{a}{\rightarrow}\left(\mathbf{d}\left(F_{a}^{-1} x\right), s\right), \text { if } x \in W_{a} \& s \in\{-, 0,+\} \\
& (x, s) \stackrel{a}{\rightarrow}\left(\mathbf{d}\left(F_{a}^{-1} x\right),-\right), \text { if } x=r\left(W_{a}\right) \& s \in\{-, 0\} \\
& (x, s) \stackrel{a}{\rightarrow}\left(\mathbf{d}\left(F_{a}^{-1} x\right),+\right), \text { if } x=l\left(W_{a}\right) \& s \in\{0,+\}
\end{aligned}
$$

If $F$ is an integer Möbius number system and $u . v \in \mathbb{P}_{A} \cap \mathbb{X}_{F}$ is a periodic word, then $\Phi(u . v)$ is either rational or quadratic. This follows from the fact that $\Phi(. v)$ satisfies the quadratic equation $F_{v}(\Phi(. v))=$ $\Phi(. v)$.

Proposition 6 If $\mathcal{W}$ is an almost-cover and $\left(F, \Sigma_{\mathcal{W}}\right)$ is a Möbius number system such that $\Phi\left([u] \cap \mathcal{S}_{\mathcal{W}}\right)=$ $\overline{W_{u}}$ for each $u \in \mathcal{L}\left(\mathcal{S}_{\mathcal{W}}\right)$, then for each $x \in \overline{\mathbb{Q}}$ there exists an infinite path with source $(x, 0)$. A word $u \in A^{\mathbb{N}}$ is the label of such a path iff $u \in \mathbb{X}_{F}$ and $\Phi(u)=x$.

Proof: Let $u$ be the label of a path with source $(x, 0)$ and denote by $x_{n}=F_{u_{[0, n)}^{-1}}(x)$, so $x_{n} \in \overline{W_{u_{n}}}$. If $x_{i}=l\left(W_{u_{i}}\right)$ for some $i$, then $x_{j} \neq r\left(W_{u_{j}}\right)$ for all $j>i$. It follows that $u \in \mathcal{S}_{\mathcal{W}}$. Since $x \in \overline{W_{u_{[0, n)}}}$ for each $n$, we get $\Phi(u)=x$. Conversely, if $\Phi(u)=x$, then $x \in \overline{W_{u_{[0, n)}}}$ for each $n$. Since $u \in \mathcal{S}_{\mathcal{W}}$, there exists a path with source $(x, 0)$ and label $u$.

Definition 7 The rational expansion interval of an $M T M \in \mathcal{M}$ is defined by

$$
\mathbf{R}(M)=\left\{x \in \overline{\mathbb{R}}:\left(M^{-1}\right)^{\bullet}(x)>\operatorname{det}(M)\right\}
$$

Note that if $x \in \overline{\mathbf{R}(M)}$ and $y=M^{-1} x$, then $\|y\| \leq\|x\|$.

Proposition 8 For each $M \in \mathcal{M}$ we have either $\mathbf{R}(M) \subseteq(0, \infty)$ or $\mathbf{R}(M) \subseteq(\infty, 0)$, and $\mathbf{R}(M)$ is nonempty iff $\|M\|>\operatorname{det}(M)+\operatorname{det}(M)^{-1}$. Moreover we have the symmetries $\mathbf{R}\left(M_{(c, d,-a,-b)}\right)=$ $\mathbf{R}\left(M_{(a, b, c, d)}\right), \mathbf{R}\left(M_{(d,-c,-b, a)}\right)=-1 / \mathbf{R}\left(M_{(a, b, c, d)}\right), \mathbf{R}\left(M_{(a,-b,-c, d)}\right)=-\mathbf{R}\left(M_{(a, b, c, d)}\right), \mathbf{R}\left(M_{(d, c, b, a)}\right)=$ $1 / \mathbf{R}\left(M_{(a, b, c, d)}\right)$, where $-1 /(x, y)=(-1 / x,-1 / y),-(x, y)=(-y,-x), 1 /(x, y)=(1 / y, 1 / x)$.

Proof: If $M=M_{(a, b, c, d)}$, then $M^{\bullet}(0)=\operatorname{det}(M) /\left(b^{2}+d^{2}\right) \leq \operatorname{det}(M)$ and $M^{\bullet}(\infty)=\operatorname{det}(M) /\left(a^{2}+c^{2}\right) \leq$ $\operatorname{det}(M)$ so neither 0 nor $\infty$ belongs to $\mathbf{R}(M)$. The rest follows from the formula for $M^{\bullet}(x)$. 
Theorem 9 Let $F: A^{*} \times \overline{\mathbb{R}} \rightarrow \overline{\mathbb{R}}$ be an integer Möbius iterative system. If $B \subset A^{+}$is a finite set such that $\mathcal{W}=\left\{\mathbf{R}\left(\left(\mathfrak{d}\left(F_{u}\right)\right): u \in B\right\}\right.$ is an almost-cover of $\overline{\mathbb{R}}$, then $\left(F, \mathcal{S}_{\mathcal{W}, A}\right)$ is a rational Möbius number system. In particular, if $\mathcal{R}=\left\{\mathbf{R}\left(F_{a}\right): a \in A\right\}$ is an almost-cover, then $\left(F, \mathcal{S}_{\mathcal{R}}\right)$ is a rational Möbius number system.

Proof: Modify the rational expansion graph of Definition 5 so that we have only edges $(x, s) \stackrel{u}{\rightarrow}(y, t)$ labelled by $u \in B$ provided $x \in \overline{\mathbf{R}\left(\mathfrak{d} F_{u}\right)}$ (and analogous conditions as in Definition 5 when $x$ is an endpoint of $\left.\mathbf{R}\left(\mathfrak{d} F_{u}\right)\right)$. In this case $\|y\|=\left\|\mathbf{d}\left(F_{u}^{-1} x\right)\right\| \leq\left\|F_{u}^{-1} x\right\| \leq\|x\|$. Since there is only a finite number of $y \in \overline{\mathbb{Q}}$ with $\|y\| \leq\|x\|$, there exists a periodic word $u . v$ such that we get a path $(x, t) \stackrel{u}{\rightarrow}(y, s) \stackrel{v}{\rightarrow}(y, s)$, so $u . v \in \mathbb{X}_{F}$ and $\Phi(u . v)=x$. Since $\mathbf{R}(M) \subseteq \mathbf{V}(M),\left(F, \mathcal{S}_{\mathcal{W}, A}\right)$ is a rational Möbius number system.

\section{$5 \quad$ Residue classes}

To show that some integer Möbius number system are not rational, we consider dynamics of integer Möbius iterative system in residue classes, which captures the cancellation of rationals during expansion. Given a positive integer $m$, we have the ring $\mathbb{Z}_{m}=\{x \in \mathbb{Z}: 0 \leq x<m\}$ of residue classes modulo $m$, and the map $\mathbf{m}_{m}: \mathbb{Z}^{2} \rightarrow \mathbb{Z}_{m}^{2}$ given by $\mathbf{m}_{m}(x)=\frac{x_{0} \bmod m}{x_{1} \bmod m}$. Denote by $\mathbb{Q}_{m}=\left\{x \in \mathbb{Z}_{m}^{2}: \operatorname{gcd}\left(x_{0}, x_{1}, m\right)=1\right\}$ and $\overline{\mathbb{Q}}_{m}=\mathbb{Q}_{m} \cup\left\{\frac{0}{0}\right\}$. For each integer matrix $M \in \mathcal{M}$ and $m>0$, the composition $\mathbf{m}_{m} M: \mathbb{Z}_{m}^{2} \stackrel{M}{\rightarrow}$ $\mathbb{Z}^{2} \stackrel{\mathfrak{m}_{m}}{\rightarrow} \mathbb{Z}_{m}^{2}$ acts on $\mathbb{Z}_{m}^{2}$.

Definition 10 Given an integer Möbius iterative system $F$ and $m>0$, we define the residue expansion graph $\mathcal{G}_{m}$ of $F$, whose vertices are $\left|\mathcal{G}_{m}\right|=\mathbb{Z}_{m}^{2}$, and whose labelled edges are $x \stackrel{a}{\rightarrow} \mathbf{m}_{m}\left(F_{a}^{-1} x\right)$.

Proposition 11 If $M \in \mathcal{M}, p$ is a prime and $k>0$ is such that $p^{k}$ does not divide $\operatorname{det}(M)$, then $\mathbf{m}_{p^{k}} M\left(\mathbb{Q}_{p^{k}}\right) \subseteq \mathbb{Q}_{p^{k}}$, i.e., if $x \in \mathbb{Q}_{p^{k}}$, then $\mathbf{m}_{p^{k}}(M x) \neq \frac{0}{0}$.

Proof: Let $M=M_{(a, b, c, d)}$ and assume that $p^{k}$ does not divide $a d-b c$. For $x \in \mathbb{Q}_{p^{k}}$ set $y=M x$ and assume that $p^{k}$ divides both $y_{0}=a x_{0}+b x_{1}$ and $y_{1}=c x_{0}+d x_{1}$. Then $p^{k}$ divides $\left(a x_{0}+b x_{1}\right) d-\left(c x_{0}+\right.$ $\left.d x_{1}\right) b=(a d-b c) x_{0}$, so $p$ divides $x_{0}$. Similarly $p^{k}$ divides $-\left(a x_{0}+b x_{1}\right) c+\left(c x_{0}+d x_{1}\right) a=(a d-b c) x_{1}$, so $p$ divides $x_{1}$ and this is a contradiction. Thus $\mathbf{m}_{p^{k}}(y) \neq \frac{0}{0}$.

Proposition 11 says that in the rational expansion graph, the rationals are cancellated only by those primes which divide some $\operatorname{det}\left(F_{a}\right)$. If $(x, s) \stackrel{a}{\rightarrow}\left(\mathbf{d}\left(F_{a}^{-1} x\right), t\right)$, and $m=\operatorname{gcd}\left(F_{a}^{-1} x\right)$, then each prime $p$ which divides $m$, divides some $\operatorname{det}\left(F_{a}\right)$, and then, $\mathbf{m}_{p}(x) \stackrel{a}{\rightarrow} \frac{0}{0}$ in $\mathcal{G}_{p}$. On the other hand, if $\mathbf{m}_{p}(x) \stackrel{a}{\rightarrow} y(p) \neq \frac{0}{0}$ for each prime $p$ which divides some $\operatorname{det}\left(F_{a}\right)$, then $\operatorname{gcd}\left(F_{a}^{-1} x\right)=1$, so we have $(x, s) \stackrel{a}{\rightarrow}\left(F_{a}^{-1}(x), t\right)$ in the rational expansion graph.

Theorem 12 Let $(F, \Sigma)$ be an integer Möbius number system such that for each a we have $\mathbf{R}\left(F_{a}\right)=\emptyset$, and $F_{a}$ is not a rotation. Assume that for each prime $p$ which divides some $\operatorname{det}\left(F_{a}\right)$ there exists a labelled graph $G_{p}$ with vertices $\left|G_{p}\right| \subseteq \mathbb{Q}_{p}$ and labelled edges $x \stackrel{a}{\rightarrow} \mathbf{m}_{p}\left(F_{a}^{-1} x\right)$, such that $\Sigma_{G_{p}} \subseteq \Sigma$. Then $(F, \Sigma)$ is not rational.

Proof: Since $F_{a}$ is not rotation, the maximum of the function $\left(F_{a}^{-1}\right)^{\bullet}(x)$ is attained at a unique point $x(a) \in \overline{\mathbb{R}}$ and $\left\|F_{a}^{-1} x\right\|>\|x\|$ for $x \neq x(a)$. There exists a point $x \in \overline{\mathbb{Q}}$ such that $\|x\|>\max \{\|x(a)\|$ : $a \in A, x(a) \in \overline{\mathbb{Q}}\}$ and moreover, for each prime $p$ which divides some $\operatorname{det}\left(F_{a}\right)$ we have $\mathbf{m}_{p}(x) \in\left|G_{p}\right|$. Then in any expansion $(x, t)=\left(x^{(0)}, t_{0}\right) \stackrel{u_{0}}{\rightarrow}\left(x^{(1)}, t_{1}\right) \stackrel{u_{1}}{\rightarrow}\left(x^{(2)}, t_{2}\right) \cdots$ no cancellation ever occurs, so we have $x^{(j+1)}=F_{u_{j}}^{-1}\left(x^{(j)}\right) \in \overline{\mathbb{Q}}$, and $\left\|x^{(0)}\right\|<\left\|x^{(1)}\right\|<\left\|x^{(2)}\right\|<\cdots$. Thus $u$ cannot be periodic.

\section{Hyperbolic rectangle systems}

Definition 13 We say that $(F, \Sigma)$ is a hyperbolic rectangle system with quotients $p, q>1$ (in horizontal and vertical directions), if its alphabet is $A=\{\overline{1}, 0,1, \overline{0}\}$ and the transformations are

$$
F_{\overline{1}}(x)=\frac{(q+1) x+(1-q)}{(1-q) x+(q+1)}, F_{0}(x)=\frac{x}{p}, F_{1}(x)=\frac{(q+1) x+(q-1)}{(q-1) x+(q+1)}, F_{\overline{0}}(x)=p x
$$

If $p=q$, we say that $(F, \Sigma)$ is a hyperbolic square system. 

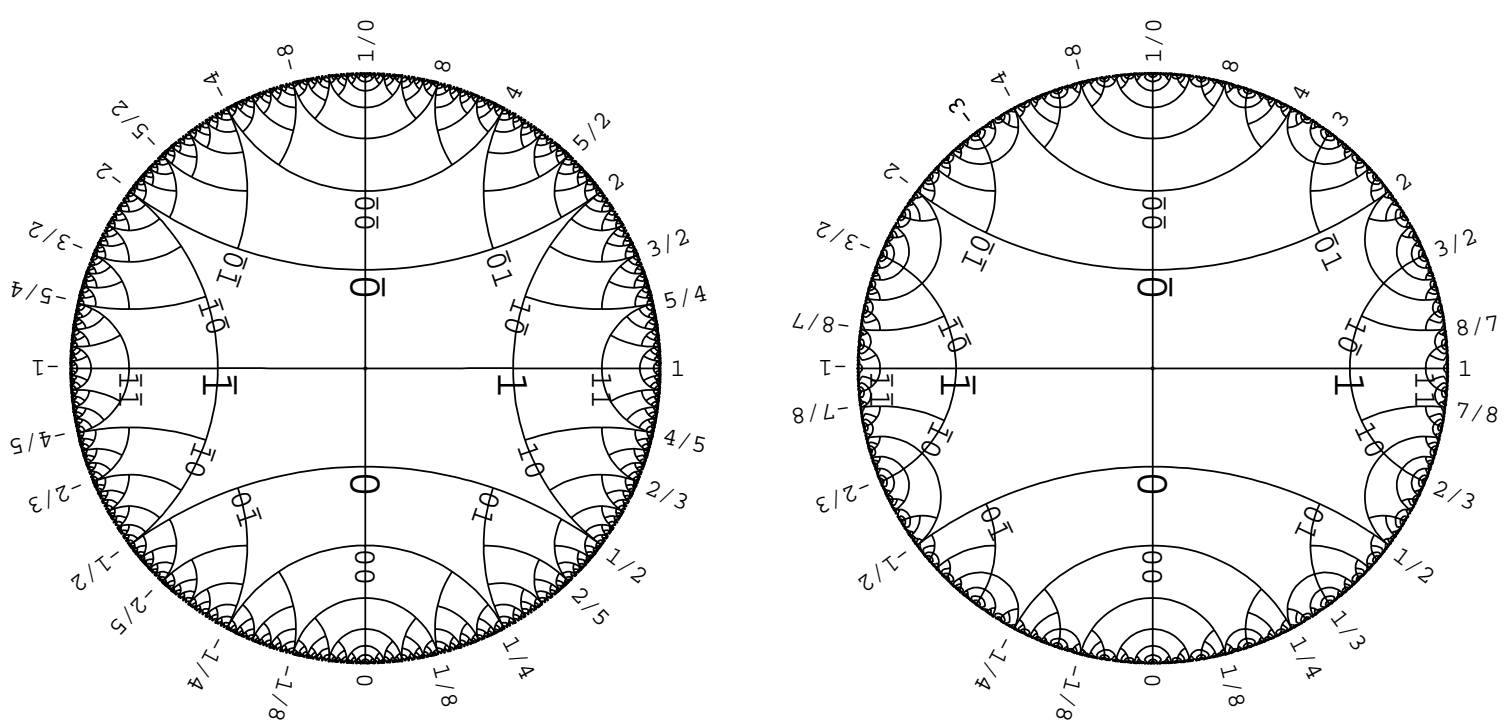

Figure 1: The means $\widehat{F}_{a}(0)$ of the biternary rectangle system with $(p, q)=(2,3)$ (left) and the biquintary rectangle system with $(p, q)=(2,5)$ (right)

Thus all $F_{a}$ are hyperbolic, the stable and unstable fixed points are $s\left(F_{\overline{1}}\right)=u\left(F_{1}\right)=-1, s\left(F_{0}\right)=$ $u\left(F_{\overline{0}}\right)=0, s\left(F_{1}\right)=u\left(F_{\overline{1}}\right)=1, s\left(F_{\overline{0}}\right)=u\left(F_{0}\right)=\infty$. The expansion quotients are $\mathbf{Q}\left(F_{0}\right)=\mathbf{Q}\left(F_{\overline{0}}\right)=p$, $\mathbf{Q}\left(F_{\overline{1}}\right)=\mathbf{Q}\left(F_{1}\right)=q$, and the expansion intervals are

$$
\mathbf{V}\left(F_{0}\right)=\left(-\frac{1}{\sqrt{p}}, \frac{1}{\sqrt{p}}\right), \mathbf{V}\left(F_{1}\right)=\left(\frac{\sqrt{q}-1}{\sqrt{q}+1}, \frac{\sqrt{q}+1}{\sqrt{q}-1}\right),
$$

$\mathbf{V}\left(F_{\overline{1}}\right)=-\mathbf{V}\left(F_{1}\right), \mathbf{V}\left(F_{\overline{0}}\right)=1 / \mathbf{V}\left(F_{0}\right)$. The expansion intervals almost-cover $\overline{\mathbb{R}}$ iff $\sqrt{p q} \leq \sqrt{p}+\sqrt{q}+1$, and the system is redundant, provided this inequality is strict. A particularly interesting case is $(p, q)=(4,9)$, when $F_{a}$ generate a Fuchsian group with noncompact fundamental domain (see Beardon [1]). If $p, q \in \mathbb{Q}$, then we get an integer Möbius iterative system with matrices in $\mathcal{M}$

$$
F_{\overline{1}}=\left[\begin{array}{rr}
r_{0} & -r_{1} \\
-r_{1} & r_{0}
\end{array}\right], F_{0}=\left[\begin{array}{rr}
p_{1} & 0 \\
0 & p_{0}
\end{array}\right], F_{1}=\left[\begin{array}{rr}
r_{0} & r_{1} \\
r_{1} & r_{0}
\end{array}\right], F_{\overline{0}}=\left[\begin{array}{rr}
p_{0} & 0 \\
0 & p_{1}
\end{array}\right]
$$

where $r_{0}=q_{0}+q_{1}, r_{1}=q_{0}-q_{1}$ if $2 \mid q_{0} q_{1}$, and $r_{0}=\left(q_{0}+q_{1}\right) / 2, r_{1}=\left(q_{0}-q_{1}\right) / 2$ if both $q_{0}$ and $q_{1}$ are odd. We have $\mathbf{R}\left(F_{a}\right)=\emptyset$ for each $a \in A$. Since $F_{\overline{1}}=F_{1}^{-1}$ and $F_{\overline{0}}=F_{0}^{-1}$ a subshift $\Sigma$ such that $(F, \Sigma)$ is a Möbius number system should have forbidden words $D=\{\overline{1} 1,0 \overline{0}, 1 \overline{1}, \overline{0} 0\}$.

Proposition 14 Let $F$ be a hyperbolic square system with rational $p=q$. If $m$ is an odd prime which divides either $q_{0}$ or $q_{1}$, then there exists a subgraph $G_{m}$ of $\mathcal{G}_{m}$ with $\left|G_{m}\right| \subseteq \mathbb{Q}_{m}$, whose subshift $\Sigma_{G_{m}}$ has forbidden words $D=\{\overline{1} 1,0 \overline{0}, 1 \overline{1}, \overline{0} 0\}$.
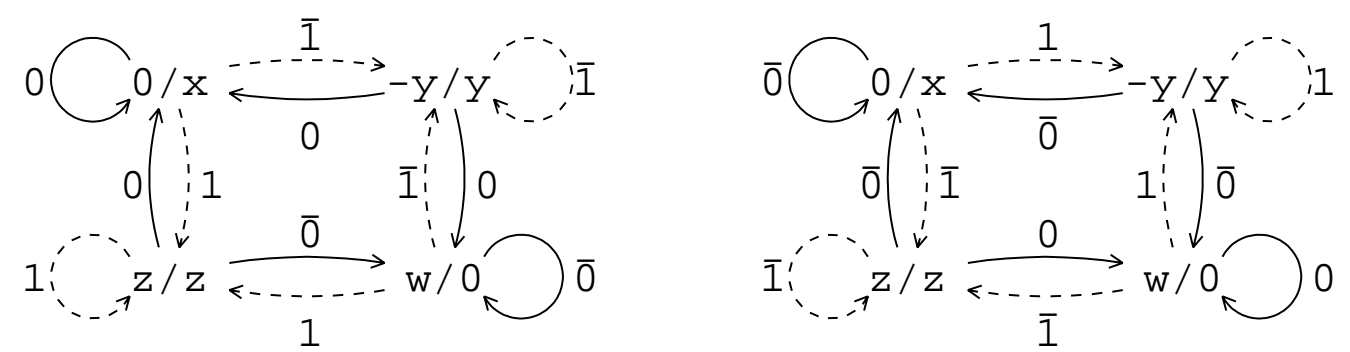

Figure 2: The graphs for $\Sigma_{D}$. The edges are full for $a \in\{\overline{1}, 1\}$ and dashed for $a \in\{0, \overline{0}\}$.

Proof: We have either $r_{0}=q_{0}+q_{1}, r_{1}=q_{0}-q_{1}$ if $2 \mid q_{0} q_{1}$, and $r_{0}=\left(q_{0}+q_{1}\right) / 2, r_{1}=\left(q_{0}-q_{1}\right) / 2$ if both $q_{0}$ and $q_{1}$ are odd. In either case we have $\operatorname{gcd}\left(r_{0}, q_{0}\right)=\operatorname{gcd}\left(r_{1}, q_{0}\right)=\operatorname{gcd}\left(q_{1}, q_{0}\right)=1$, $\operatorname{so} \operatorname{gcd}\left(r_{0}, m\right)=$ $\operatorname{gcd}\left(r_{1}, m\right)=1$. Define the vertices of $G_{m}$ by

$$
\left|G_{m}\right|=\left\{x \in \mathbb{Q}_{m}: x_{0}=0 \vee x_{1}=0 \vee x_{0}=x_{1} \vee x_{0}+x_{1}=0\right\},
$$


where the equalities are modulo $m$. Consider labelled edges

$$
\begin{array}{rlllllllllll}
\frac{0}{x} & \stackrel{\overline{1}}{\rightarrow} & \frac{r_{1} x}{r_{0} x}, & \frac{0}{x} & \stackrel{0}{\rightarrow} & \frac{0}{q_{1} x}, & \frac{0}{x} & \stackrel{1}{\rightarrow} & \frac{-r_{1} x}{r_{0} x}, & \frac{0}{x} & \stackrel{\overline{0}}{\rightarrow} & \frac{0}{q_{0} x}, \\
\frac{x}{x} & \stackrel{\overline{1}}{\rightarrow} & \frac{\left(r_{0}+r_{1}\right) x}{\left(r_{0}+r_{1}\right) x}, & \frac{x}{x} & \stackrel{0}{\rightarrow} & \frac{q_{0} x}{q_{1} x}, & \frac{x}{x} & \stackrel{1}{\rightarrow} & \frac{\left(r_{0}-r_{1}\right) x}{\left(r_{0}-r_{1}\right) x}, & \frac{x}{x} & \stackrel{\overline{0}}{\rightarrow} & \frac{q_{1} x}{q_{0} x}, \\
\frac{x}{0} & \stackrel{\overline{1}}{\rightarrow} & \frac{r_{0} x}{r_{1} x}, & \frac{x}{0} & \stackrel{0}{\rightarrow} & \frac{q_{0} x}{0}, & \frac{x}{0} & \stackrel{1}{\rightarrow} & \frac{r_{0} x}{-r_{1} x}, & \frac{x}{0} & \stackrel{\overline{0}}{\rightarrow} & \frac{q_{1} x}{0}, \\
\frac{-x}{x} & \stackrel{\overline{1}}{\rightarrow} & \frac{\left(r_{1}-r_{0}\right) x}{\left(r_{0}-r_{1}\right) x}, & \frac{-x}{x} & \stackrel{0}{\rightarrow} & \frac{-q_{0} x}{q_{1} x}, & \frac{-x}{x} & \stackrel{1}{\rightarrow} & \frac{-\left(r_{0}+r_{1}\right) x}{-\left(r_{0}+r_{1}\right) x}, & \frac{-x}{x} & \stackrel{\overline{0}}{\rightarrow} & \frac{-q_{1} x}{q_{0} x},
\end{array}
$$

If $m$ divides $q_{0}$ then $m$ divides also $r_{0}+r_{1}, \operatorname{gcd}\left(m, q_{1}\right)=\operatorname{gcd}\left(m, r_{0}-r_{1}\right)=1$, and we take as edges of $G_{m}$ only those which occur in the graph in Figure 2 left. For example, the last edge in the first row $\frac{0}{x} \stackrel{\overline{0}}{\rightarrow} \frac{0}{q_{0} x}=\frac{0}{0}$ is excluded from $G_{m}$. It is easy to see that the subshift of the graph is $\Sigma_{G_{m}}=\Sigma_{D}$. If $m$ divides $q_{1}$ then $m$ divides also $r_{0}-r_{1}, \operatorname{gcd}\left(m, q_{0}\right)=\operatorname{gcd}\left(m, r_{0}+r_{1}\right)=1$, and we take as edges of $G_{m}$ only those which occur in the graph in Figure 2 right. We get again $\Sigma_{G_{m}}=\Sigma_{D}$ (see Figure 3 bottom left and right).
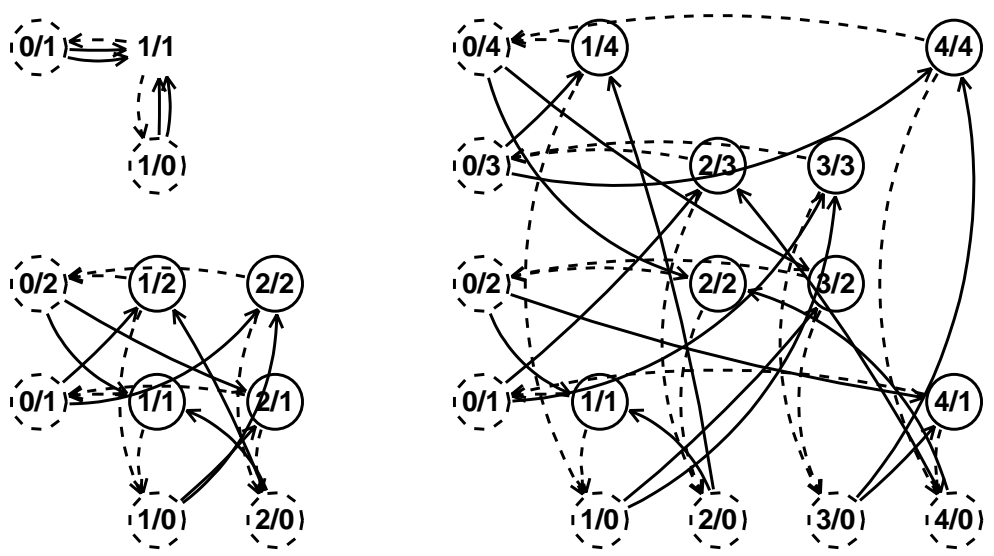

Figure 3: Subgraphs of the residue graphs for the hyperbolic square system with $p=q=2$ (upper left), $p=q=3$ (bottom left) and $p=q=5$ (right). The edges are full for $a \in\{\overline{1}, 1\}$ and dashed for $a \in\{0, \overline{0}\}$. A self-edge $x \stackrel{a}{\rightarrow} x$ is indicated by the circle around $x$.

Proposition 15 Let $F$ be a hyperbolic square system with rational $p=q$ and assume that 2 divides $q_{0} q_{1}$. Then there exists a subgraph $G_{2}$ of $\mathcal{G}_{2}$ with vertices $\left|G_{2}\right|=\mathbb{Q}_{2}$, whose subshift $\Sigma_{G_{2}}$ has forbidden words $D^{\prime}=\{\overline{1} 1,0 \overline{0}, 1 \overline{1}, \overline{0} 0, \overline{11}, 11\}$.

Proof: The edges of $G_{2}$ are (see Figure 3 top left)

$$
\begin{aligned}
& \frac{0}{1} \stackrel{\overline{1}}{\rightarrow} \frac{1}{1}, \frac{0}{1} \stackrel{0}{\rightarrow} \frac{0}{1}, \frac{0}{1} \stackrel{1}{\rightarrow} \frac{1}{1}, \frac{1}{1} \stackrel{0}{\rightarrow} \frac{0}{1}, \frac{1}{1} \stackrel{\overline{0}}{\rightarrow} \frac{1}{0}, \frac{1}{0} \stackrel{\overline{1}}{\rightarrow} \frac{1}{1}, \frac{1}{0} \stackrel{\frac{1}{\rightarrow}}{\frac{1}{1}}, \frac{1}{0} \stackrel{\overline{0}}{\rightarrow} \frac{1}{0} \quad \text { if } \quad \mathbf{m}_{2}(q)=\frac{0}{1}, \\
& \frac{0}{1} \stackrel{1}{\rightarrow} \frac{1}{1}, \frac{0}{1} \frac{1}{\rightarrow} \frac{1}{1}, \frac{0}{1} \stackrel{\overline{0}}{\rightarrow} \frac{0}{1}, \frac{1}{1} \stackrel{0}{\rightarrow} \frac{1}{0}, \frac{1}{1} \stackrel{0}{\rightarrow} \frac{0}{1} \frac{1}{0} \stackrel{\overline{1}}{\rightarrow} \frac{1}{1}, \frac{1}{0} \stackrel{0}{\rightarrow} \frac{1}{0}, \frac{1}{0} \stackrel{\frac{1}{\rightarrow}}{\rightarrow} \quad \text { if } \quad \mathbf{m}_{2}(q)=\frac{1}{0} .
\end{aligned}
$$

Theorem 16 No integer hyperbolic square system $(F, \Sigma)$ with $\Sigma \subseteq \Sigma_{\{\overline{1} 1,0 \overline{0}, 1 \overline{1}, \overline{0} 0\}}$ is rational.

Proof: It is easy to see that $\mathbf{R}\left(F_{a}\right)=\emptyset$ for all $a \in A$. If both $q_{0}$ and $q_{1}$ are odd, the claim follows from Theorem 12 and Proposition 14. Assume now that $q_{0} q_{1}$ is even, so $r_{0}=q_{0}+q_{1}, r_{1}=q_{0}-q_{1}$. For $n>1$, the product matrices $F_{\overline{1}^{n}}=\left(F_{\overline{1}}\right)^{n}, F_{1^{n}}=\left(F_{1}\right)^{n}$ have all entries even. After cancellation we get matrices with odd entries

$$
\mathfrak{d} F_{\overline{1}^{n}}=\left[\begin{array}{cc}
q_{0}^{n}+q_{1}^{n} & q_{1}^{n}-q_{0}^{n} \\
q_{1}^{n}-q_{0}^{n} & q_{0}^{n}+q_{1}^{n}
\end{array}\right], \mathfrak{d} F_{1^{n}}=\left[\begin{array}{cc}
q_{0}^{n}+q_{1}^{n} & q_{0}^{n}-q_{1}^{n} \\
q_{0}^{n}-q_{1}^{n} & q_{0}^{n}+q_{1}^{n}
\end{array}\right]
$$

and the rational expansion intervals of these matrices are empty as well. We modify the expansion graph so that the multiplication by $n$ consecutive $F_{\overline{1}}$ or $F_{1}$ are replaced by multiplication by $\mathfrak{d} F_{\overline{1}}^{n}$ or $\mathfrak{d} F_{1}^{n}$. In the 


\begin{tabular}{|l|l|l||l|l|l|}
\hline$u$ & $F_{u}$ & $\mathbf{R}\left(\mathfrak{d} F_{u}\right)$ & $u$ & $F_{u}$ & $\mathbf{R}\left(\mathfrak{d} F_{u}\right)$ \\
\hline$\overline{0100} 1$ & $(30,12,-6,0)$ & $\left(\frac{1}{0},-\frac{14}{5}\right)$ & $\overline{010}$ & $(8,-2,-2,2)$ & $(-8,-2)$ \\
$\overline{101}$ & $(9,-6,-6,6)$ & $\left(-2,-\frac{6}{7}\right)$ & $\overline{1} 0 \overline{1}$ & $(6,-6,-6,9)$ & $\left(-\frac{7}{6},-\frac{1}{2}\right)$ \\
$0 \overline{1} 0$ & $(2,-2,-2,8)$ & $\left(-\frac{1}{2},-\frac{1}{8}\right)$ & $0 \overline{1} 001$ & $(0,-6,12,30)$ & $\left(-\frac{5}{14}, 0\right)$ \\
$0100 \overline{1}$ & $(0,6,-12,30)$ & $\left(0, \frac{5}{14}\right)$ & 010 & $(2,2,2,8)$ & $\left(\frac{1}{2}, \frac{1}{2}\right)$ \\
101 & $(6,6,6,9)$ & $\left(\frac{1}{2}, \frac{7}{6}\right)$ & $1 \overline{0} 1$ & $(9,6,6,6)$ & $\left(\frac{6}{7}, 2\right)$ \\
$\overline{0} 1 \overline{0}$ & $(8,2,2,2)$ & $(2,8)$ & $\overline{0} 1 \overline{001}$ & $(30,-12,6,0)$ & $\left(\frac{14}{5}, \frac{1}{0}\right)$ \\
\hline
\end{tabular}

Table 1: Rational expansion intervals of the biternary rectangle system

corresponding residue 2 expansion graph, all matrices $\mathfrak{d} F_{\overline{1}}^{n}$ and $\mathfrak{d} F_{1}^{n}$ are represented by the same matrix $(1,1,1,1)$. Thus we can represent words $\overline{1}^{n}$ and $1^{n}$ by letters $\overline{1}$ and 1 , so that a path in the modified expansion graph on $\overline{\mathbb{Q}}$ maps to a path with forbidden words $D^{\prime}=\{\overline{1} 1,0 \overline{0}, 1 \overline{1}, \overline{0} 0, \overline{11}, 11\}$. By Proposition 15 , it follows that if $\mathbf{d}_{m}(x) \in\left|G_{m}\right|$ for all primes $p$ which $\operatorname{divide}$ some $\operatorname{det}\left(F_{a}\right)$, then in the modified expansion graph no cancellation ever occurs, and the norm $\|x\|$ increases during the expansion of $x$, so the expansion of $x$ cannot be periodic.

Proposition 17 The hyperbolic rectangle systems with parameters $(2,3),(3,2),(4,3),(3,4),(2,5),(5,2)$ are rational.

Proof: In each case, a finite set $B \subset A^{+}$can be found such that $\left\{\mathbf{R}\left(\mathfrak{d} F_{u}\right): u \in B\right\}$ is an almost-cover of $\overline{\mathbb{R}}$. In the case of biternary system with $(p, q)=(2,3)$ such a cover is given in Table 1 .

In Figure 1 we show the biternary system with $(p, q)=(2,3)$ (left) and the biquintary system with $(p, q)=(2,5)$ (right), both with the interval partition $\mathcal{W}=\left(\left(-2,-\frac{1}{2}\right),\left(-\frac{1}{2}, \frac{1}{2}\right),\left(\frac{1}{2}, 2\right),(2,-2)\right)$. In the case of biternary system we get a sofic subshift $\mathcal{S}_{\mathcal{W}}=\{\overline{1}, 0\}^{\mathbb{N}} \cup\{0,1\}^{\mathbb{N}} \cup\{1, \overline{0}\}^{\mathbb{N}} \cup\{\overline{0}, \overline{1}\}^{\mathbb{N}}$ which is a union of four full shifts, one for each quadrant: $\Phi\left(\{0,1\}^{\mathbb{N}}\right)=[0,1], \Phi\left(\{1, \overline{0}\}^{\mathbb{N}}\right)=[1, \infty], \Phi\left(\{\overline{0}, \overline{1}\}^{\mathbb{N}}\right)=[\infty,-1]$, $\Phi\left(\{\overline{1}, 0\}^{\mathbb{N}}\right)=[-1,0]$. Figure 1 shows the means $\widehat{F}_{u}(0)$ of words $u \in \mathcal{L}\left(\mathcal{S}_{\mathcal{W}}\right)$ in the unit disc $\mathbb{D}$. The curves between these means are constructed as follows. For each MT $M$ there exists a family of MT $\left(M^{t}\right)_{t \in \mathbb{R}}$ such that $M^{0}=\mathrm{Id}, M^{1}=M$, and $M^{t+s}=M^{t} M^{s}$. In Figure 1, each mean $\widehat{F}_{u}(0)$ is joined to $\widehat{F}_{u a}(0)$ by the curve $\left(\widehat{F}_{u} \widehat{F}_{a}^{t}(0)\right)_{0 \leq t \leq 1}$. The labels $u \in A^{+}$at $\widehat{F}_{u}(0)$ are written in the direction of the tangent vectors $\widehat{F}_{u}^{\prime}(0)$.

\section{Modular systems}

We say that $(F, \Sigma)$ is a modular Möbius number system if it consists of modular transformations $F_{a} \in \mathcal{M}_{1}$ with unit determinant. In modular systems we have $\mathbf{V}\left(F_{a}\right)=\mathbf{R}\left(F_{a}\right)$, so the condition for the convergence of Theorem 3 coincides with the condition for rationality of Theorem 9 . Since $\mathbf{R}\left(F_{a}\right)$ is included either in $(0, \infty)$ or $(\infty, 0)$, the condition for redundancy from Theorems 3 and 4 never applies.

Theorem 18 If $\left(F, \mathcal{S}_{\mathcal{W}}\right)$ is a modular Möbius number system such that $\Phi\left([u] \cap \mathcal{S}_{\mathcal{W}}\right)=\overline{W_{u}}$ for each $u \in \mathcal{L}\left(\mathcal{S}_{\mathcal{W}}\right)$, then $\mathbf{Q}(\mathcal{W})=1$ and the system is not redundant.

Proof: By Theorem 4 we have $\mathbf{Q}(\mathcal{W}) \geq 1$. By Proposition 8,0 and $\infty$ do not belong to any $\mathbf{R}\left(F_{a}\right)$, so $\mathbf{Q}(\mathcal{W}) \leq 1$, and therefore $\mathbf{Q}(\mathcal{W})=1$. Since $\Phi: \mathcal{S}_{\mathcal{W}} \rightarrow \overline{\mathbb{R}}$ is continuous, by compactness there exists $u \in \mathcal{S}_{\mathcal{W}}$, such that for each $n>0$ the set $\Phi\left(\left[u_{[0, n)}\right]\right)$ contains an interval, whose closure contains $\Phi(u)$. Denote by $a:=\Phi(u)$ and consider a continuous function $g: \overline{\mathbb{R}} \rightarrow \overline{\mathbb{R}}$, which on a neighbourhood of $a$ has the form $g(x)=(x-a) \sin 1 /(x-a)$, so $g \Phi\left(\left[u_{[0, n)}\right]\right)$ contains 0 as an inner point. Let $f: \mathcal{S}_{\mathcal{W}} \rightarrow \mathcal{S}_{\mathcal{W}}$ be continuous function with $\Phi f=g \Phi$. By Proposition 8, for each $u \in \mathcal{L}(\Sigma)$ we have either $W_{u} \subseteq(0, \infty)$ or $W_{u} \subseteq(\infty, 0)$, so for each $n$ there exists $k$ such that $g \Phi\left(\left[u_{[0, n)}\right]\right) \subseteq \Phi\left(\left[f(u)_{[0, k)}\right]\right) \subseteq \overline{W_{f(u)_{[0, k)}}}$ which is included either in $[0, \infty]$ or in $[\infty, 0]$. This is a contradiction.

Modular systems are closely connected to continued fractions. In Kưrka [6, 4] we give examples of modular systems with transformations $F_{\overline{1}}(x)=x-1, F_{0}=-1 / x$, and $F_{1}(x)=x+1$. We have $\mathbf{R}\left(F_{\overline{1}}\right)=\left(\infty,-\frac{1}{2}\right), \mathbf{R}\left(F_{1}\right)=\left(\frac{1}{2}, \infty\right)$, but $\mathbf{R}\left(F_{0}\right)=\emptyset$, since $F_{0}$ is a rotation. In the present context we 

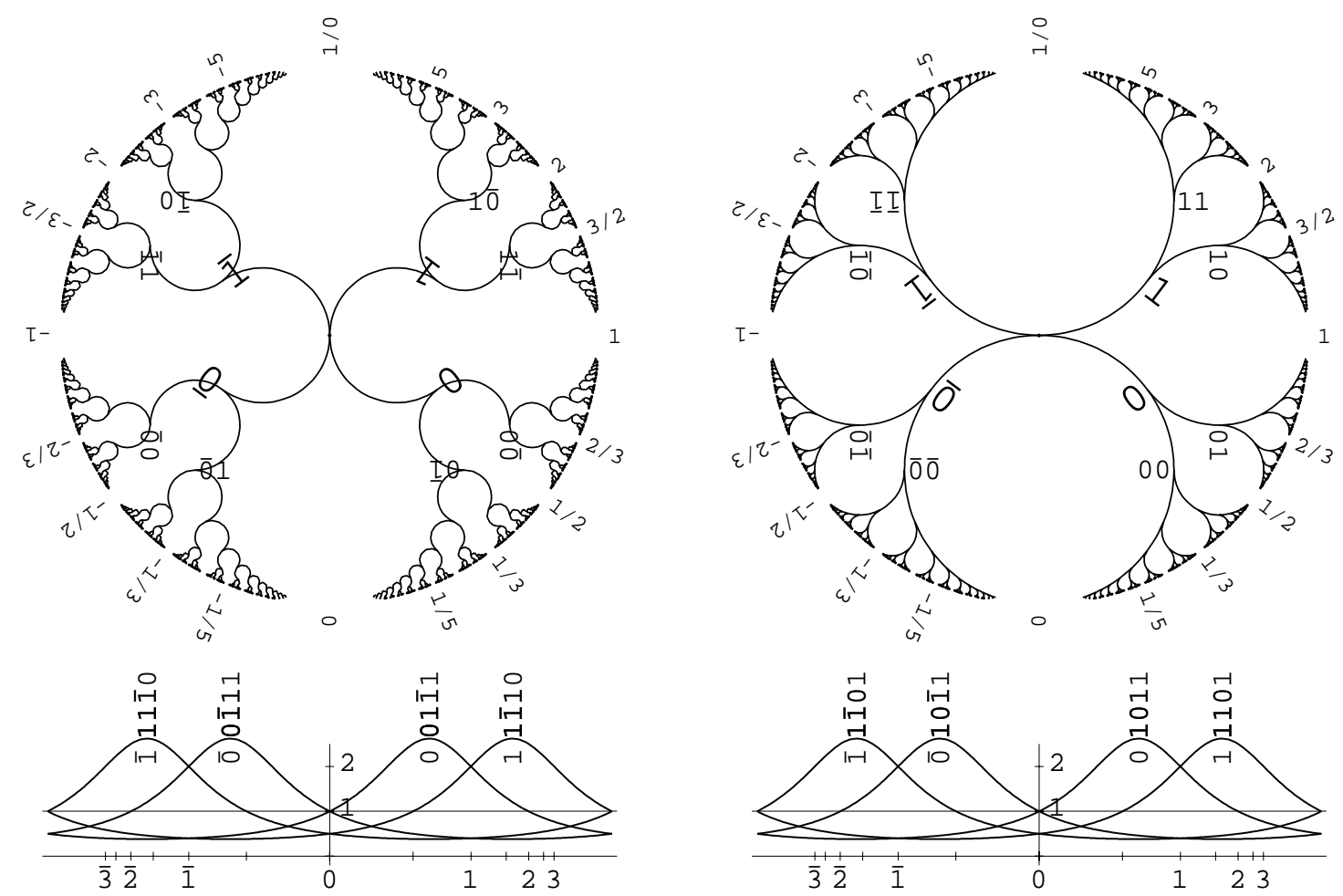

Figure 4: The elliptic (left) and the parabolic (right) modular systems: means $\widehat{F}_{a}(0)$ (top) and circle derivations $\left(F_{a}^{-1}\right)^{\bullet}(x)$ (bottom)

\begin{tabular}{|c|c|c|c||c|c|c|c|}
\hline$a$ & $F_{a}$ & $\mathbf{R}\left(F_{a}\right)$ & $F_{a}^{-1}\left(W_{a}\right)$ & $a$ & $F_{a}$ & $\mathbf{R}\left(F_{a}\right)$ & $F_{a}^{-1}\left(W_{a}\right)$ \\
\hline$\overline{1}$ & $(1,1,-1,0)$ & $\left(\infty,-\frac{1}{2}\right)$ & $(0, \infty)$ & $\overline{1}$ & $(1,-1,0,1)$ & $\left(\infty,-\frac{1}{2}\right)$ & $(\infty, 0)$ \\
$\overline{0}$ & $(0,-1,1,1)$ & $(-2,0)$ & $(0, \infty)$ & $\overline{0}$ & $(1,0,-1,1)$ & $(-2,0)$ & $(\infty, 0)$ \\
0 & $(0,1,-1,1)$ & $(0,2)$ & $(\infty, 0)$ & 0 & $(1,0,1,1)$ & $(0,2)$ & $(0, \infty)$ \\
1 & $(1,-1,1,0)$ & $\left(\frac{1}{2}, \infty\right)$ & $(\infty, 0)$ & 1 & $(1,1,0,1)$ & $\left(\frac{1}{2}, \infty\right)$ & $(0, \infty)$ \\
\hline$\overline{1}$ & $(2,1,-1,0)$ & $(\infty,-1)$ & $(0,-1)$ & $\overline{1}$ & $(1,-2,0,1)$ & $(\infty,-1)$ & $(\infty, 1)$ \\
$\overline{0}$ & $(0,-1,1,2)$ & $(-1,0)$ & $(-1, \infty)$ & $\overline{0}$ & $(1,0,-2,1)$ & $(-1,0)$ & $(1,0)$ \\
0 & $(0,1,-1,2)$ & $(0,1)$ & $(\infty, 1)$ & 0 & $(1,0,2,1)$ & $(0,1)$ & $(0,-1)$ \\
1 & $(2,-1,1,0)$ & $(1, \infty)$ & $(1,0)$ & 1 & $(1,2,0,1)$ & $(1, \infty)$ & $(-1, \infty)$ \\
\hline
\end{tabular}

Table 2: Modular transformations

consider rather systems whose transformations have the same expansion quotients and the same lengths of their expansion intervals.

Example 1 The elliptic modular system consists of the alphabet $A=\{\overline{1}, \overline{0}, 0,1\}$, transformations with matrices

$$
F_{\overline{1}}=\left[\begin{array}{rr}
1 & 1 \\
-1 & 0
\end{array}\right], F_{\overline{0}}=\left[\begin{array}{rr}
0 & -1 \\
1 & 1
\end{array}\right], F_{0}=\left[\begin{array}{rr}
0 & 1 \\
-1 & 1
\end{array}\right], F_{1}=\left[\begin{array}{rr}
1 & -1 \\
1 & 0
\end{array}\right],
$$

and the interval partition $\mathcal{W}$ with $W_{\overline{1}}=(\infty,-1), W_{\overline{0}}=(-1,0), W_{0}=(0,1), W_{1}=(1, \infty)$.

All $F_{a}$ are elliptic with $F_{a}^{3}=\mathrm{Id}$, and $F_{\overline{10}}=F_{10}=$ Id. The rational expansion intervals and the preimages of $W_{a}$ can be seen in Table 2 top left. We see that $\mathcal{W}$ satisfies the condition of Proposition 2, so $\mathcal{S}_{\mathcal{W}}$ is a SFT of order 2 with forbidden words $00,01,10,11, \overline{11}, \overline{10}, \overline{01}, \overline{00}$. Thus $u \in \mathcal{L}\left(\mathcal{S}_{\mathcal{W}}\right)$ iff consecutive letters $u_{i}, u_{i+1}$ belong to different subalphabets $A_{-}=\{\overline{1}, \overline{0}\}, A_{+}=\{0,1\}$. In Figure 4 we show the means (top left) and the circle derivations (bottom left) of all $F_{a}^{-1}$ evaluated at $x=\tan \frac{t}{2}=\mathbf{d}^{-1}\left(e^{i t}\right)$ for $t \in(-\pi, \pi)$.

Example 2 The parabolic modular system consists of the alphabet $A=\{\overline{1}, \overline{0}, 0,1\}$, transformations

$$
F_{\overline{1}}=\left[\begin{array}{rr}
1 & -1 \\
0 & 1
\end{array}\right], F_{\overline{0}}=\left[\begin{array}{rr}
1 & 0 \\
-1 & 1
\end{array}\right], F_{0}=\left[\begin{array}{ll}
1 & 0 \\
1 & 1
\end{array}\right], F_{1}=\left[\begin{array}{ll}
1 & 1 \\
0 & 1
\end{array}\right],
$$




\begin{tabular}{|l|l||l|l||l|l||l|l||l|l|}
\hline $1 / 4$ & 0000.1 & $1 / 3$ & 000.1 & $2 / 5$ & 0010.1 & $1 / 2$ & 00.1 & $3 / 5$ & 0100.1 \\
$2 / 3$ & 010.1 & $3 / 4$ & 0110.1 & $1 / 1$ & 0.1 & $4 / 3$ & 1000.1 & $3 / 2$ & 100.1 \\
$5 / 3$ & 1010.1 & $2 / 1$ & 10.1 & $5 / 2$ & 1100.1 & $3 / 1$ & 110.1 & $4 / 1$ & 1110.1 \\
\hline
\end{tabular}
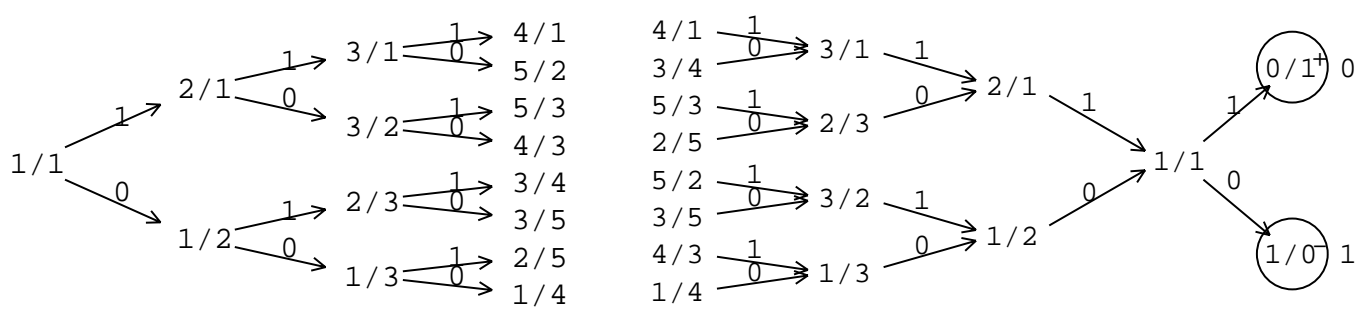

Figure 5: The Stern-Brocot tree (left) and the expansions of Farey fractions in the parabolic modular system (right). The vertex $(x, s)$ of the expansion graph is written as $x^{s}$ provided $s \neq 0$. A self-edge $(x, s) \stackrel{a}{\rightarrow}(x, s)$ is indicated by a circle around $(x, s)$.

and the interval partition $\mathcal{W}$ with $W_{\overline{1}}=(\infty,-1), W_{\overline{0}}=(-1,0), W_{0}=(0,1), W_{1}=(1, \infty)$

All $F_{a}$ are parabolic with fixed points $\infty$ or 0 , and $F_{\overline{1} 1}=F_{\overline{0} 0}=$ Id. Because $\mathbf{R}\left(M_{(a, b, c, d)}\right)=\mathbf{R}\left(M_{(c, d,-a,-b)}\right)$, the system has the same rational expansion intervals as the elliptic modular system of Example 1. The subshift $\mathcal{S}_{\mathcal{W}}=\{0,1\}^{\mathbb{N}} \cup\{\overline{1}, \overline{0}\}^{\mathbb{N}}$ is of finite type. Negative numbers have expansions in the alphabet $A_{-}=\{\overline{1}, \overline{0}\}$ and positive numbers have expansions in the alphabet $A_{+}=\{0,1\}$ The parabolic modular system expands rational numbers according to the Stern-Brocot tree, which systematically enumerates Farey fractions (see Figure 5). If $u \in A_{+}^{*}$ is the address of $x$ in the Stern-Brocot tree, then $x$ has exactly two expansions $u 0.1$ and $u 1.0$. This is proved in M.Niqui [9]. There are two more modular systems whose transformations are given in Table 2 bottom left and bottom right. In both these systems the rational expansion intervals coincide with the intervals of $\mathcal{W}$, and their subshifts $\mathcal{S}_{\mathcal{W}}$ are of order two with forbidden words $D=\left\{u \in A^{2}: F_{u}=\mathrm{Id}\right\}$.

\section{Bimodular systems}

Besides being nonredundant, modular systems are slowly converging since they have the expansion quotient $\mathbf{Q}(\mathcal{W})=1$. This is why nonmodular systems are more convenient for expansions of numbers and for arithmetical algorithms. If $F: A^{*} \times \overline{\mathbb{R}} \rightarrow \overline{\mathbb{R}}$ is an integer Möbius iterative system such that no $F_{a}$ is modular and if $\mathcal{R}=\left\{\mathbf{R}\left(F_{a}\right): a \in A\right\}$ is an almost-cover of $\overline{\mathbb{R}}$, then $\mathcal{V}=\left\{\mathbf{V}\left(F_{a}\right): a \in A\right\}$ is a cover, since $\mathbf{R}\left(F_{a}\right) \subset \operatorname{int} \mathbf{V}\left(F_{a}\right)$, so $\left(F, \mathcal{S}_{\mathcal{V}}\right)$ is a redundant Möbius number system. If we require $\operatorname{det}\left(F_{a}\right)>1$ for all transformations of the system, then we need at least eight transformations.

Proposition 19 For $k=1,2,3$ the rational expansion intervals of the following eight matrices almostcover $\overline{\mathbb{R}}$ :

$$
\left[\begin{array}{rr}
1 & 0 \\
\pm k & 2
\end{array}\right],\left[\begin{array}{rr}
2 & 0 \\
\pm k & 1
\end{array}\right],\left[\begin{array}{rr}
1 & \pm k \\
0 & 2
\end{array}\right],\left[\begin{array}{rr}
2 & \pm k \\
0 & 1
\end{array}\right]
$$

Proof: The endpoints of $\mathbf{R}\left(M_{(1,0, k, 2)}\right)$ are 0 and $2 k /\left(k^{2}+3\right)$, the endpoints of $\mathbf{R}\left(M_{(2,0, k, 1)}\right)$ are $1 / k$ and $3 / k$, the endpoints of $\mathbf{R}\left(M_{(1, k, 0,2)}\right)$ are $k / 3$ and $k$, the endpoints of $\mathbf{R}\left(M_{(2, k, 0,1)}\right)$ are $\left(k^{2}+3\right) / 2 k$ and $\infty$,

The order of the rational expansion intervals (or of points where the maxima of the circle derivation are attained) of the transformations from Proposition 19 depends on $k$. In all three cases we use the same alphabet $A=\{\overline{3}, \overline{2}, \overline{1}, \overline{0}, 0,1,2,3\}$ and the canonical partition $\mathcal{W}=\left(W_{a}\right)_{a \in A}$ with $W_{\overline{3}}=(\infty,-2)$, $W_{\overline{2}}=(-2,-1), W_{\overline{1}}=\left(-1,-\frac{1}{2}\right), W_{\overline{0}}=\left(-\frac{1}{2}, 0\right), W_{0}=\left(0, \frac{1}{2}\right), W_{1}=\left(\frac{1}{2}, 1\right), W_{2}=(1,2), W_{3}=(2, \infty)$.

Definition 20 For $k=1,2,3$, the bimodular $k$-octanic system $\left(F, \mathcal{S}_{\mathcal{W}}\right)$ consists of the alphabet $A=\{\overline{3}, \overline{2}, \overline{1}, \overline{0}, 0,1,2,3\}$, the canonical partition $\mathcal{W}$ and the transformations from Proposition 19 whose assignments to the alphabet $A$ are in Figures 6 and $\%$. 

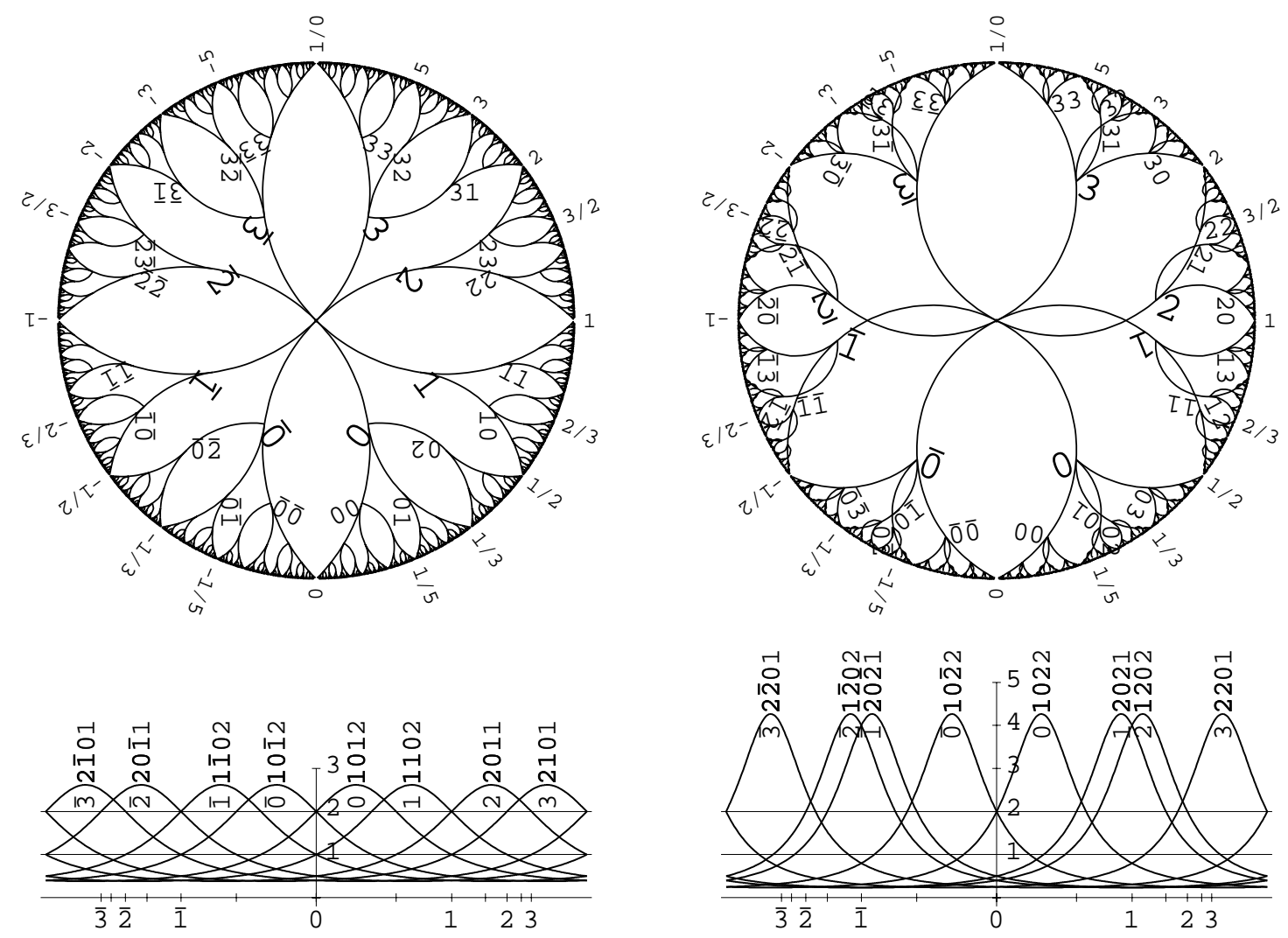

Figure 6: The bimodular $k$-octanic systems with $k=1$ (left) and $k=2$ (right): means(top) and circle derivations (bottom)

In all three cases, $\mathcal{W}$ satisfies the conditions of Proposition 2, so $\mathcal{S}_{\mathcal{W}}$ is a SFT of order 2. For $k=1,2$ the positive numbers can be expressed in the alphabet $A_{+}=\{0,1,2,3\}$, and negative numbers in the alphabet $A_{-}=\{\overline{3}, \overline{2}, \overline{1}, \overline{0}\}$. In both cases, the subshift can be written as a disjoint union $\mathcal{S}_{\mathcal{W}}=$ $\left(\Sigma_{\mathcal{W}} \cap A_{-}^{\mathbb{N}}\right) \cup\left(\Sigma_{\mathcal{W}} \cap A_{+}^{\mathbb{N}}\right)$ with forbidden words of the positive part $D=\{03,12,13,20,21,30\}$ for $k=1$ and $D=\{10,23\}$ for $k=2$. Expansions of Farey fractions in these systems can be seen in Table 3. The expansion graph for the 2-octanic system is in Figure 8. All three systems have symmetries $n, r, n r: A \rightarrow A$, and $i_{k}: A \rightarrow A$ which depends on $k$ :

\begin{tabular}{|c|cccccccc|}
\hline & $\overline{3}$ & $\overline{2}$ & $\overline{1}$ & $\overline{0}$ & 0 & 1 & 2 & 3 \\
\hline$i_{1}$ & 1 & 0 & 3 & 2 & $\overline{2}$ & $\overline{3}$ & $\overline{0}$ & $\overline{1}$ \\
$i_{2}, i_{3}$ & 2 & 3 & 0 & 1 & $\overline{1}$ & $\overline{0}$ & $\overline{3}$ & $\overline{2}$ \\
$n$ & 3 & 2 & 1 & 0 & $\overline{0}$ & $\overline{1}$ & $\overline{2}$ & $\overline{3}$ \\
$r$ & $\overline{0}$ & $\overline{1}$ & $\overline{2}$ & $\overline{3}$ & 3 & 2 & 1 & 0 \\
$n r$ & 0 & 1 & 2 & 3 & $\overline{3}$ & $\overline{2}$ & $\overline{1}$ & $\overline{0}$ \\
\hline
\end{tabular}

Then $F_{i(a)}=F_{a}^{-1}, W_{n(a)}=-W_{a}, W_{r(a)}=1 / W_{a}$, and $W_{n r(a)}=-1 / W_{a}$. It follows that if $\Phi(u)=x$, then $\Phi(n(u))=-x, \Phi(r(u))=1 / x$, and $\Phi(n r(u))=-1 / x$. All transformations $F_{a}$ are hyperbolic with stable fixed points $\infty,-k,-1 / k, 0,0,1 / k, k, \infty$.

Besides these three systems $\left(F, \mathcal{S}_{\mathcal{W}}\right)$ with the canonical partition $\mathcal{W}$, we may consider the systems $\left(F, \mathcal{S}_{\mathcal{R}}\right)$ with the rational expansion almost-cover $\mathcal{R}=\left(\mathbf{R}\left(F_{a}\right)\right)_{a \in A}$, in which the rational numbers have more expansions, possibly shorter than in the canonical systems. For all three systems we have $\mathbf{Q}(\mathcal{W})=$ $\mathbf{Q}(\mathcal{R})=2$ but $\mathbf{L}(\mathcal{W})=\mathbf{L}(\mathcal{R})=0$, so neither of these systems is redundant. We get redundant systems $\left(F, \mathcal{S}_{\mathcal{V}}\right)$ with the expansion intervals $\mathcal{V}=\left(\mathbf{V}\left(F_{a}\right)\right)_{a \in A}$ (see Figure 9 ). For $k=1$ we have $\mathbf{Q}(\mathcal{V})=1$, $\mathbf{L}(\mathcal{V})=1.287$ and $\mathcal{V}$ has rational endpoints $0, \pm \frac{1}{3}, \pm \frac{1}{2}, \pm 1, \pm 2, \pm 3, \infty$. For $k=2$ we have $\mathbf{Q}(\mathcal{V})=$ $1.24, \mathbf{L}(\mathcal{V})=0.77$ and for $k=3$ we get $\mathbf{Q}(\mathcal{V})=1.35, \mathbf{L}(\mathcal{V})=0.53$, but these covers do not have rational endpoints. Nevertheless there are many interval covers with rational endpoints, positive Lebesgue numbers and expansion quotients greater than one, and any of these covers would be suitable for the arithmetical algorithms. 

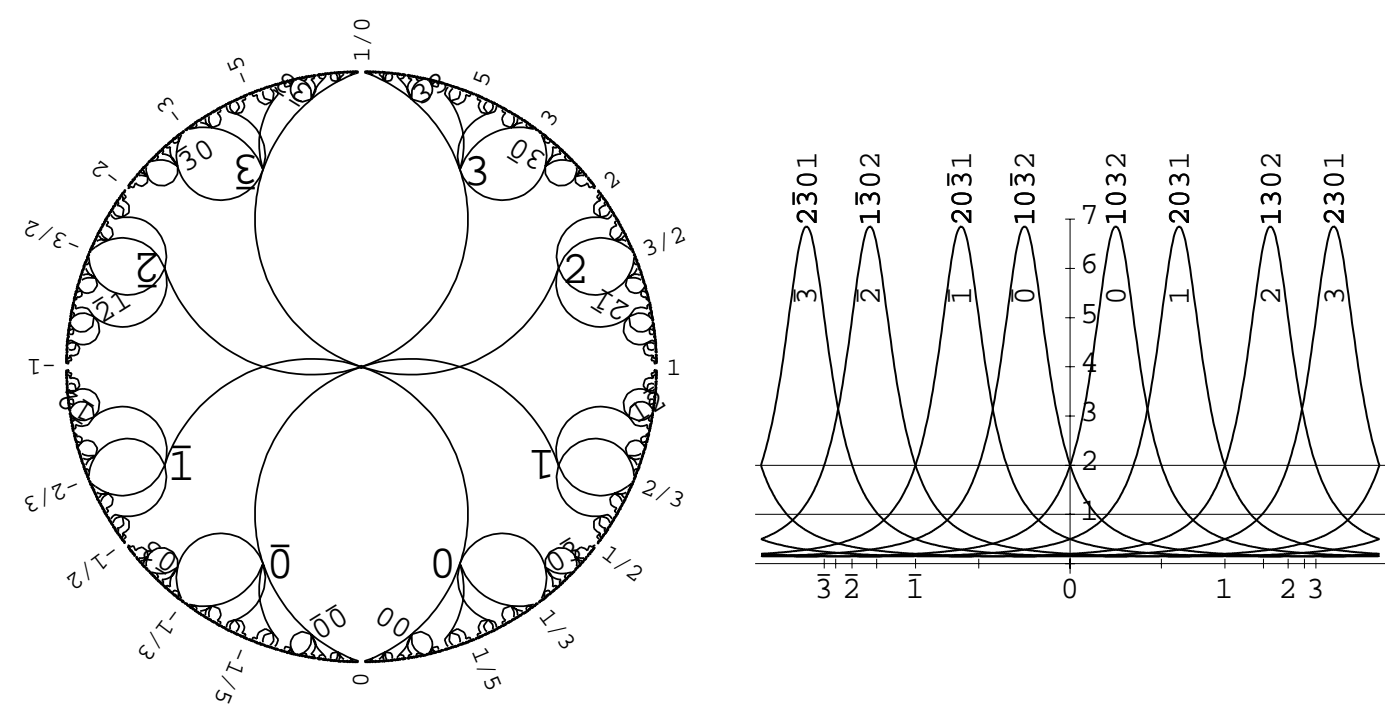

Figure 7: The bimodular 3-octanic system: means(left) and circle derivations (right)

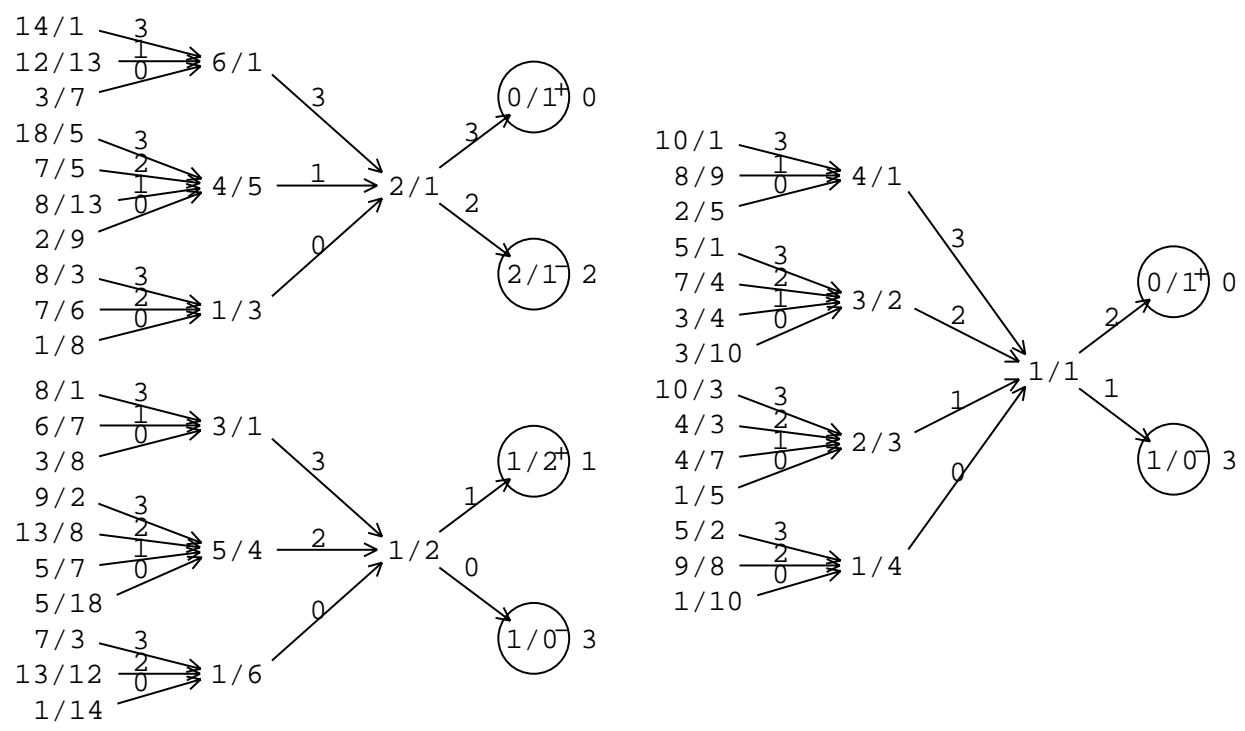

Figure 8: The rational expansion graph of the bimodular 2-octanic system. The vertex $(x, s)$ is written as $x^{s}$ provided $s \neq 0$. A self-edge $(x, s) \stackrel{a}{\rightarrow}(x, s)$ is indicated by a circle around $(x, s)$.

\section{Acknowledgments}

The research was supported by the Research Program CTS MSM 0021620845 and by the Czech Science Foundation research project GAČR 201/09/0854. A part of this research has been done during my stay in the Max-Planck-Institut für Mathematik in Bonn.

\section{References}

[1] A. F. Beardon. The geometry of discrete groups. Springer-Verlag, Berlin, 1995.

[2] A. Kazda. Convergence in Möbius number systems. Integers, 2:261-279, 2009.

[3] P. Kůrka. A symbolic representation of the real Möbius group. Nonlinearity, 21:613-623, 2008.

[4] P. Kůrka. Geometry of Möbius number systems. Technical Report MPIM 2009-59, Max-PlanckInstitut für Mathematik, Bonn, 2009. 


\begin{tabular}{|c|c|c|c|c|c|c|c|c|c|}
\hline $1 / 4$ & 0101.1 & $1 / 3$ & 01.1 & $2 / 5$ & 022.3 & $1 / 2$ & 1.0 & $3 / 5$ & 101.0 \\
\hline $2 / 3$ & 101.1 & $3 / 4$ & 11.0 & $1 / 1$ & 1.1 & $4 / 3$ & 22.3 & $3 / 2$ & 231.1 \\
\hline $5 / 3$ & 232.3 & $2 / 1$ & 2.3 & $5 / 2$ & 311.0 & $3 / 1$ & 31.1 & $4 / 1$ & 3231.1 \\
\hline $1 / 4$ & $\overline{01.3}$ & $1 / 3$ & $\overline{02.2}$ & $2 / 5$ & $\begin{array}{l}031.3 \\
\end{array}$ & $1 / 2$ & 0.3 & $3 / 5$ & 1121. \\
\hline $2 / 3$ & 11.3 & $3 / 4$ & 121.3 & $1 / 1$ & 1.3 & $4 / 3$ & 211.3 & $3 / 2$ & 21.3 \\
\hline $5 / 3$ & 2211.3 & $2 / 1$ & 2.2 & $5 / 2$ & 301.3 & $3 / 1$ & 30.3 & $4 / 1$ & 31.3 \\
\hline $1 / 4$ & $02.1 \overline{2}$ & $1 / 3$ & 03.3 & $2 / 5$ & $0 \overline{31} . \overline{2} 1$ & $1 / 2$ & $0 . \overline{3} 0$ & $3 / 5$ & 130.0 \\
\hline $2 / 3$ & 13.3 & $3 / 4$ & $1 \overline{3} 0.0$ & $1 / 1$ & $1 . \overline{2} 1$ & $4 / 3$ & $2 \overline{0} 3.3$ & $3 / 2$ & 20.0 \\
\hline $5 / 3$ & 203.3 & $2 / 1$ & $2.1 \overline{2}$ & $5 / 2$ & $3 \overline{03} .0 \overline{3}$ & $3 / 1$ & 30.0 & $4 / 1$ & $30 . \overline{3} 0$ \\
\hline
\end{tabular}

Table 3: Expansions of Farey fractions in the $k$-modular octanic systems $k=1$ (top) $k=2$ (center), $k=3$ (bottom)
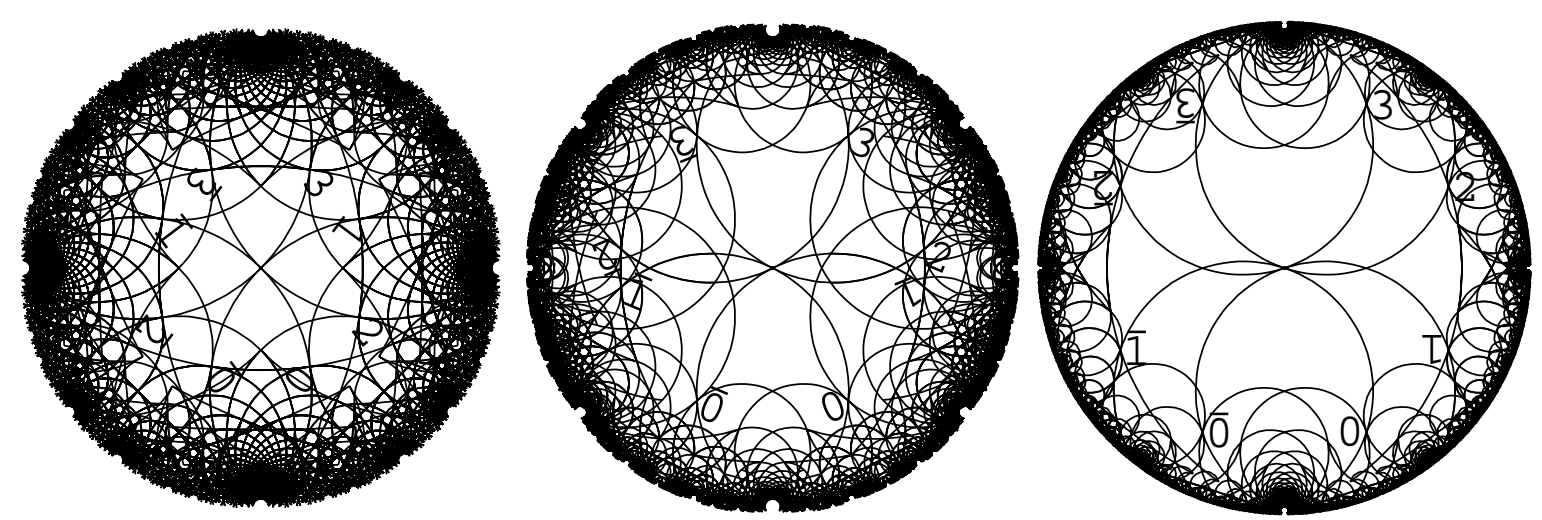

Figure 9: Redundant bimodular octanic $k$-systems $\left(F, \mathcal{S}_{\mathcal{V}}\right)$ with $k=1$ (left), $k=2$ (center) and $k=3$ (right).

[5] P. Kůrka. Iterative systems of real Möbius transformations. Discrete and Continuous Dynamical Systems, 25(2):567-574, 2009.

[6] P. Kůrka. Möbius number systems with sofic subshifts. Nonlinearity, 22:437-456, 2009.

[7] P. Kůrka and A.Kazda. Möbius number systems based on interval covers. 2009. submitted.

[8] D. Lind and B. Marcus. An Introduction to Symbolic Dynamics and Coding. Cambridge University Press, Cambridge, 1995.

[9] M. Niqui. Exact real arithmetic on the Stern-Brocot tree. J. Discrete Algorithms, 5(2):356-379, 2007.

[10] J. E. Vuillemin. Exact real computer arithmetic with continued fractions. IEEE Transactions on Computers, 39(8):1087-1105, August 1990. 